Research Article: New Research / Cognition and Behavior

\title{
Personalized connectome-based modeling in patients with semi-acute phase TBI: relationship to acute neuroimaging and 6- month follow-up
}

https://doi.org/10.1523/ENEURO.0075-21.2022

Cite as: eNeuro 2022; 10.1523/ENEURO.0075-21.2022

Received: 22 February 2021

Revised: 10 January 2022

Accepted: 14 January 2022

This Early Release article has been peer-reviewed and accepted, but has not been through the composition and copyediting processes. The final version may differ slightly in style or formatting and will contain links to any extended data.

Alerts: Sign up at www.eneuro.org/alerts to receive customized email alerts when the fully formatted version of this article is published. 4.0 International license, which permits unrestricted use, distribution and reproduction in any medium provided that the original work is properly attributed. 
1. Manuscript Title: Personalized connectome-based modeling in patients with semi-acute phase TBI: relationship to acute neuroimaging and 6-month follow-up

2. Abbreviated Title: Brain simulations of early-phase TBI

3. Author Names and Affiliations Tyler Gooda,b, Michael Schirnerc,d,e, Kelly Shena, Petra Ritterc,d,e, Pratik Mukherjee ${ }^{\mathrm{f}, \mathrm{g}, \mathrm{h}}$, Brian Levinea ${ }^{\mathrm{a}, \mathrm{b}}$, Anthony Randal McIntosha,b

${ }^{a}$ Rotman Research Institute, Baycrest Health Sciences, 3560 Bathurst St, Toronto, Ontario M6A 2E1, Canada

${ }^{\mathrm{b}}$ University of Toronto, 27 King's College Cir, Toronto, Ontario M5S, Canada

${ }^{c}$ Charité - Universitätsmedizin Berlin, corporate member of Freie Universität Berlin, Humboldt-Universität zu Berlin, and Berlin Institute of Health, Dept. of Neurology, Germany

${ }^{\mathrm{d}}$ Bernstein Focus State Dependencies of Learning \& Bernstein Center for Computational Neuroscience, Berlin, Germany

${ }^{\mathrm{e}}$ Einstein Center for Neuroscience Berlin, Charitéplatz 1, 10117 Berlin

${ }^{\mathrm{f}}$ Einstein Center Digital Future, Wilhelmstraße 67, 10117 Berlin $^{\mathrm{f}}$ Department of Radiology \& Biomedical Imaging, UCSF, San Francisco, CA, USA

${ }^{g}$ Brain and Spinal Cord Injury Center, Zuckerberg San Francisco General Hospital and Trauma Center, San Francisco, CA, USA

${ }^{\mathrm{h}}$ Department of Bioengineering and Therapeutic Sciences, University of California, San Francisco, San Francisco, CA, USA

4. Author Contributions: TG performed research, analyzed data, wrote paper; MS analyzed data, wrote paper; KS analyzed data, wrote paper; PR wrote paper, analyzed data; PM designed research, wrote paper; BL designed research, analyzed data, wrote paper; ARM analyzed data, wrote paper

5. Correspondence should be addressed to: 3560 Bathurst Street, Toronto, Ontario M6A 2E1. Tel.: 416-785-2500 ext 3522;

rmcintosh@research.baycrest.org

6. Number of Figures: 8

7. Number of Tables: 6

8. Number of Multimedia: 0

9. Number of words for abstract: 248 
10. Number of words for Significance Statement: 107

11. Number of words for Introduction: 740

12. Number of words for Discussion: 1319

\section{Acknowledgements}

The research herein was supported by the National Science and Engineering Research Council, the James S. McDonnell Foundation, the Canadian Institutes of Health Research, the Ontario Neurotrauma Foundation, the Alzheimer's Society of Canada, Berlin Institute of Health \& Foundation Charité, H2020

Research and Innovation Action and ERC grants, German Research Foundation.

\section{Conflict of Interests}

a. The authors report no conflict of interest

\section{Funding sources:}

a. Postgraduate Scholarship (doctoral) from the National Science and Engineering Research Council (NSERC) awarded to T.J.G.

b. NSERC grant (RGPIN-2017-06793) to A.R.M.

c. Canadian Institutes of Health Research Catalyst (CIHR; Grant \# CBT 127060) awarded to B.L.

d. Ontario Neurotrauma Foundation (Grant \# 2012-ABI-CAT3-973) awarded to B.L.

e. CIHR Operating Grant (Grant \# MOP133728) to B.L.

f. H2020 Research and Innovation Action grants 826421 (Virtual Brain Cloud), 785907 (Human Brain Project) and ERC 683049 awarded to P.R.

g. German Research Foundation CRC 1315 \& 936 and grant RI 2073/6-1 to P.R.

h. Berlin Institute of Health \& Foundation Charité, Johanna Quandt Excellence Initiative awarded to P.R. 
Personalized connectome-based modeling in patients with semi-acute phase TBI:

relationship to acute neuroimaging and 6-month follow-up

\section{Abstract}

4 Following traumatic brain injury (TBI), cognitive impairments manifest through

5 interactions between microscopic and macroscopic changes. On the micro-scale a

6 neurometabolic cascade alters neurotransmission, while on the macro-scale diffuse

7 axonal injury impacts the integrity of long-range connections. Large-scale brain network

8 modeling allows us to make predictions across these spatial scales by integrating

9 neuroimaging data with biophysically based models to investigate how microscale

10 changes invisible to conventional neuroimaging influence large-scale brain dynamics. To

11 this end, we analyzed structural and functional neuroimaging data from a well

12 characterized sample of forty-four adult TBI patients recruited from a regional trauma

13 center, scanned at 1-2 weeks post-injury, and with follow-up behavioral outcome

14 assessed six months later. Thirty-six age-matched healthy adults served as comparison

15 participants. Using The Virtual Brain we fit simulations of whole-brain resting-state

16 functional MRI to the empirical static and dynamic functional connectivity of each

17 participant. Multivariate partial least squares (PLS) analysis showed that patients with

18 acute traumatic intracranial lesions had lower cortical regional inhibitory connection

19 strengths than comparison participants, while patients without acute lesions did not differ

20 from the comparison group. Further multivariate PLS analyses found correlations

21 between lower semi-acute regional inhibitory connection strengths and more symptoms

22 and lower cognitive performance at a 6-month follow-up. Critically, patients without

23 acute lesions drove this relationship, suggesting clinical relevance of regional inhibitory 
24 connection strengths even when traumatic intracranial lesions were not present. Our

25 results suggest large-scale connectome-based models may be sensitive to

26 pathophysiological changes in semi-acute phase TBI patients and predictive of their

27 chronic outcomes.

\section{Significance Statement}

The variability of clinical outcomes following mild to moderate traumatic brain

30 injury (TBI) is underscored by complex pathophysiological mechanisms that take effect

31 across spatial scales. We used the neuroinformatics platform, The Virtual Brain, to model

32 individualized brain activity and make inferences across these spatial scales. Specifically,

33 this approach allowed us to link macroscopic brain dynamics with mesoscopic

34 biophysical parameters, distinguishing semi-acute mild to moderate TBI patients from

35 comparison participants and predicting the long-term recovery of these patients. Our

36 results demonstrate the sensitivity of our large-scale brain model to pathophysiological

37 changes following TBI and illustrates how computational modeling may be used to

38 advance understanding of chronic TBI outcome.

\section{Introduction}

40 Chronic clinical outcomes following traumatic brain injury (TBI) are heterogeneous

41 (Dabek \& Caban, 2016; Si et al., 2018). Classifying patients based on the presence of

42 pathoanatomic features on computerized tomography (CT) and/or magnetic resonance

43 imaging (MRI) is a useful way to stratify variance of TBI patients (Iverson et al., 2012;

44 McMahon et al., 2014; Palacios et al., 2017; Yuh et al., 2014). However, even within

45 these patient subgroups significant variability in clinical outcomes and cognitive

46 performance can be observed (Iverson et al., 2012; Palacios et al., 2017). The sources of 
47 this variability are diverse (Kenzie et al., 2018) and may include forms of pathology not

48 visible with conventional neuroimaging.

49 TBI has been described as a multi-scale system deficit, with cognitive impairments

50 manifesting through interactions between microscopic and macroscopic changes (Kenzie

51 et al., 2018). On the macro-scale, TBI is associated with decreased integrity of white

52 matter pathways and an imbalance and inefficiency of functional networks (Hayes,

53 Bigler, \& Verfaellie, 2016). Studies using diffusion weighted MRI imaging (dwMRI)

54 have consistently detected decreases in fractional anisotropy (FA) (Douglas et al., 2015;

55 Filley \& Kelly, 2018; Niogi \& Mukherjee, 2010; Shenton et al., 2012) that correlate with

56 cognition (Palacios et al., 2020; Wallace, Mathias, \& Ward, 2018) and if assessed in the

57 semi-acute phase, long-term clinical outcomes (Yuh et al., 2014). Functional connections

58 are also sensitive to TBI, with alterations observed in multiple intrinsic connectivity

59 networks (for reviews: Hayes et al., 2016; Sharp, Scott, \& Leech, 2014). Early phase

60 functional connectivity (FC) between and within networks may be predictive of long-

61 term symptom severity (Madhavan et al., 2019; Palacios et al., 2017), while changes to

62 FC dynamics have been noted in the acute (Hou et al., 2019) and semi-acute phase

63 (Mayer et al., 2014; Vergara, Mayer, Kiehl, \& Calhoun, 2018) following TBI.

64 On the micro-scale, TBI causes a neurometabolic cascade that alters

65 neurotransmission and can have long-lasting effects (Giza \& Hovda, 2015). The initial

66 injury causes a sudden imbalance in glutamatergic, and GABAergic neurotransmitter

67 levels, as well as NMDA receptor malfunction (Giza \& Hovda, 2015). Magnetic

68 resonance spectroscopy (MRS) is capable of detecting changes to neuromodulatory

69 concentrations in vivo. A recent meta-analysis found evidence for elevated glutamate 
70 concentrations in adult patients after a single mild TBI (mTBI) in the acute and sub-acute

71 phases (Eisele, Hill-Strathy, Michels, \& Rauen, 2020). Furthermore, some studies have

72 shown these elevated glutamate concentrations are predictive of long-term outcomes

73 (Shutter, Tong, \& Holshouser, 2004).

74 Connectome-based brain network modeling provides a novel perspective to TBI by

75 allowing access to local and global parameters related to both micro-level

76 neuromodulatory changes and macro-level connectivity changes. We used the

77 neuroinformatics platform The Virtual Brain (TVB) to simulate whole-brain dynamics

78 composed of interacting neural population models (Ritter, Schirner, McIntosh, \& Jirsa,

79 2013; Sanz Leon et al., 2013). Our model simulated brain areas as excitatory and

80 inhibitory neural populations connected via GABA and NMDA synapses (Deco,

81 McIntosh, et al., 2014; Schirner et al., 2018). Personalized simulations were constrained

82 by each subject's structural connectome and fitted to their static and dynamic functional

83 connectomes, which were computed from dwMRI and rsfMRI (Schirner, Rothmeier,

84 Jirsa, McIntosh, \& Ritter, 2015). The parameter fitting procedure tuned the parameters

85 global coupling and regional inhibitory connection strengths to yield FC predictions for

86 each subject. Global coupling is a scaling factor related to the level of

87 integration/segregation in the system (Deco, Tononi, Boly, \& Kringelbach, 2015), while

88 regional inhibitory connection strength indicates the level of inhibitory influence at each

89 brain region. These model parameters have been used to describe healthy neural

90 dynamics (Jirsa et al., 2010; Roy et al., 2014) and those of clinical populations (Aerts et

91 al., 2018; Falcon et al., 2016; Jirsa et al., 2017; Zimmermann et al., 2018). 
In the present exploratory study we considered a well-characterized sample of

93 TBI patients and healthy comparison participants. Previous studies of this sample have

94 described group differences and predictive correlations between semi-acute phase

95 neuroimaging data and 6-month behavioural outcome measures using fractional

96 anisotropy (Yuh et al., 2014) and functional connectivity (Palacios et al., 2017). In the

97 present work we integrated these modalities with personalized TVB simulations, which

98 allowed us to consider the role of local neural dynamics in patient outcomes. We

99 examined group differences as well as predictive correlations between semi-acute local

100 brain dynamics and clinical outcomes at a six-month follow-up. Our analyses considered

101 two patient subgroups: CT/MRI positive, defined as patients with any signs of traumatic

102 intracranial lesions on day-of-injury CT scan or semi-acute MRI, and CT/MRI negative,

103 defined as patients without any such abnormality.

104

\section{Method}

\section{Participants}

Forty-four patients and thirty-six comparison participants were acquired from the

107 pilot phase of the Transforming Research and Clinical Knowledge in Traumatic Brain

108 Injury project (Track-TBI Pilot) (Yue et al., 2013). Patients were collected from

109 convenience sampling at the acute care, level I trauma center in San Francisco General

110 hospital. The inclusion criteria included CT scan to assess for evidence of acute TBI

111 within 24 hours of injury, Glasgow Coma Scale (GCS) score of 13-15 (upon emergency

112 department (ED) arrival), loss of consciousness (LOC) $<30$ minutes, post-traumatic

113 amnesia (PTA) duration $<24$ hours, and age $18-55$ years (inclusive). Patients with a 
114 previous significant TBI (LOC $>5$ mins) were also excluded. Overlapping patient and

115 comparison participant samples have been described in detail in two previous

116 publications (Palacios et al., 2017; Yuh et al., 2014). Eight patients did not complete the

117 clinical and cognitive assessment at the six-month follow-up and were therefore removed

118 from analyses using this data $\left(\mathrm{n}_{\text {patient }}=36\right.$ for six month clinical/cognitive data).

119 Each patient's head CT upon ED presentation and semi-acute brain MRI (5-18

120 days post-injury) was characterized using the TBI common data elements (TBI-CDE)

121 criteria (Yue et al., 2013). Each CT and MRI was anonymized and reviewed by a board

122 certified neuroradiologist blinded to the data. The TBI patients were divided into two

123 subgroups: 1) CT/MRI positive ( $\left.\mathrm{n}=14 ; \mathrm{M}_{\mathrm{age}}=39, \mathrm{SD}_{\mathrm{age}}=13.7\right)$, defined as patients with

124 any acute traumatic intracranial lesion (epidural hematoma [EDH], subdural hematoma

$125[\mathrm{SDH}]$, subarachnoid hemorrhage $[\mathrm{SAH}]$, contusion, or evidence of traumatic axonal

126 injury [TAI]) and/or depressed skull fracture on either CT or MRI, and 2) CT/MRI

127 negative $\left(\mathrm{n}=30 ; \mathrm{M}_{\mathrm{age}}=31, \mathrm{SD}_{\mathrm{age}}=9.0\right)$, defined as patients without any such abnormality

128 on either CT or MRI. There were no large lesions expected to adversely affect the

129 dwMRI or fMRI results. Patient characteristics are presented in Table 1. We note that all

130 the TBI patients meet the definition of mTBI by some standards (Yue et al, 2013) though

131 the patients with positive CT findings would be considered moderate by other criteria

132 (Krainin et al, 2011). 


\section{Outcome Measures}

The outcome measures included the Extended Glasgow Outcome Scale (GOS-E)

135 at 6 months post-injury performed through structured interviews with each participant by

136 research assistants trained to uniformly assess the GOS-E. A trained neuropsychologist

137 also administered the following behavioural and cognitive tests at 6 months after injury:

138 Trail Making Tests (TMT) Part A and B, Wechsler Adult Intelligence Scale (WASI), $4^{\text {th }}$

139 Ed (Wechsler, 1999), Satisfaction with Life Scale (SWLS) (Diener, Emmons, RJ, \&

140 Griffin, 1985), Brief Symptom Inventory (BSI) 18 (Derogatis, 1983), and California

141 Verbal Learning Test-Second Edition (CVLT-II) (Delis et al, 2000). Higher scores on the

142 GOS-E, SWLS, WAIS, and CVLT were coded such that higher scores indicate better

143 outcome, while scores on the BSI and TMT are coded such that higher scores indicate

144 poorer outcome.

Principal Component Analysis

Principal component analysis (PCA) was used to expose the latent structure

147 within the collection of outcome measures and to reduce the dimensionality of the

148 dataset. Data were transformed if significantly skewed $(p<0.05)$ using square root or

$149 \log 10$ to improve the correlation structure. The point of inflection on a scree plot was

150 used to identify the number of components to keep. After extracting this number of

151 factors, a promax rotation was performed using the "principal" function in R (R Core

152 Team, 2013). The correlation between factors was $r=0.33$, exceeding 0.32 and therefore

153 indicating greater than $10 \%$ overlap in variance between factors, warranting an oblique

154 rotation (Tabachnick \& Fidell, 2007). As such, we used the two-factor solution with a 
155 promax rotation for later analyses. Factors were designated as (1) TBI Symptoms, and (2)

156 Age and Cognition based on the primary symptoms, cognitive functions, and

157 characteristics assessed by the variables that strongly contributed to each (loading $>|0.3|$;

158 Table 5). Factor scores for each participant represent the degree to which they express the

159 factor. Higher factor scores on the TBI Symptoms factor indicates poorer outcome, while

160 higher factor scores on the Age and Cognition factor implies older age and lower

161 performance.

\section{Imaging Procedure}

163 MRIs were acquired on a 3T GE Signa EXCITE scanner equipped with an eight-

164 channel phased array head radiofrequency coil. The following conventional 3T MRI

165 sequences were performed: 1) axial three-dimensional (3D) inversion recovery fast

166 spoiled gradient recalled echo $\mathrm{T} 1$-weighted images $(\mathrm{TE}=1.5 \mathrm{~ms}$; $\mathrm{TR}=6.3 \mathrm{~ms}$; inversion

167 time $[\mathrm{TI}]=400 \mathrm{~ms}$; flip angle, 15 degrees) with $230 \mathrm{~mm}$ FOV, 156 contiguous partitions

$168(1.0 \mathrm{~mm})$ at $256 \cdot 256$ matrix; 2) axial T2-weighted fluid-attenuated inversion recovery

169 images $(\mathrm{TE}=126 \mathrm{~ms} ; \mathrm{TR}=10 \mathrm{sec} ; \mathrm{TI}=2200 \mathrm{~ms})$ with $220 \mathrm{~mm}$ FOV, $47-48$ contiguous

170 slices $(3.0 \mathrm{~mm})$ at $256 \cdot 256$ matrix; and 3) axial magnetization-prepared gradient echo

$171 \mathrm{~T} 2 *$ - weighted images ( $\mathrm{TE}=15 \mathrm{~ms}$; $\mathrm{TR}=500 \mathrm{~ms}$; flip angle 20 degrees $)$ with 220 .

$172170 \mathrm{~mm}$ FOV and $47-48$ contiguous slices $(3.0 \mathrm{~mm})$ at $256 \cdot 192$ matrix. A 7 min. rsfMRI

173 single shot gradient-echo echo planar imaging (EPI) sequence was acquired (repetition

174 time $[\mathrm{TR}]=2000 \mathrm{~ms}$, echo time $[\mathrm{TE}]=28 \mathrm{~ms}$; flip angle $=90$ grad; field of view $[\mathrm{FOV}]$

$175=220 \mathrm{~mm}$; voxel size $=3.4 \cdot 3.4 \cdot 4.0 \mathrm{~mm}$ ). The subjects were asked to close their eyes,

176 relax, not focus their attention on anything specific, and not fall asleep. Whole-brain DTI

177 was performed with a multi-slice single-shot spin echo echoplanar pulse sequence (echo 
178 time $[\mathrm{TE}]=63 \mathrm{~ms}$; repetition time $[\mathrm{TR}]=14 \mathrm{sec}$ ) using 55 diffusion-encoding directions,

179 isotropically distributed over the surface of a sphere with electrostatic repulsion, acquired

180 at $b=1000 \mathrm{sec} / \mathrm{mm} 2$, seven acquisitions at $b=0 \mathrm{sec} / \mathrm{mm} 2,72$ interleaved slices of 1.8 -

$181 \mathrm{~mm}$ thickness each with no gap between slices, a $128 \cdot 128$ matrix, and a field of view

182 (FOV) of $230 \cdot 230 \mathrm{~mm}$. For DTI, parallel imaging was employed using the array spatial

183 sensitivity encoding technique (ASSET) with an acceleration factor of 2. The MRI

184 scanner and the scanning protocol used were the same for the patient and comparison

185 groups.

\section{Parcellation Scheme}

The 96-region of interest (ROI) Regional Map parcellation (Kötter \& Wanke,

188 2005) was used for construction of the structural and functional connectivity matrices.

189 The RM-96 parcellation has 82 cortical and 14 subcortical ROIs (Bezgin, Solodkin,

190 Bakker, Ritter, \& Mcintosh, 2017). It has been used previously in TVB models (Ritter et

191 al., 2013; Shen, Bezgin, et al., 2019) and was developed to harmonize cytoarchitectonic,

192 topographic and functional definitions of brain regions across primate species (Kotter \&

193 Wanke, 2005), which is an advantage for network modeling work that integrates

194 structural and functional neuroimaging data.

\section{5 dwMRI Preprocessing and Tractography}

196 Preprocessing of dwMRI data, and subsequent tractography was completed using

197 a Python implementation of a previously reported procedure (Shen, Goulas, et al., 2019).

198 Eddy current induced distortions were corrected for using FSL's 'eddy_correct'

199 command, and the diffusion gradient vectors rotated accordingly. The MNI152_T1_1mm 
200 standard brain included with FSL was then registered to each subject's T1-weighted

201 image using a non-linear registration conducted with Advanced Normalization Tools

202 (ANTs). Warps produced in this step were used to map the 96-ROI Regional Map

203 parcellation (ANTs: Avants et al., 2011; RM-96 parcellation: Bezgin et al., 2017) onto

204 each subject's T1-weighted image using ANTs' 'WarpImageMultiTransform' executable

205 and a nearest neighbor interpolation. FSL's FLIRT function was used to register subject

206 T1-weighted images to dwMRI space. Seed and target ROI masks were defined as the

207 white matter (WM) voxels adjacent to each gray matter (GM) ROI within each region of

208 the RM-96 parcellation scheme. An exclusion mask for each seed mask was also created

209 using the GM voxels adjacent to the seed mask. For intrahemispheric tracking, exclusion

210 masks of the opposite hemisphere were also used. Diffusion tensor models were fitted at

211 each voxel by FSL's 'dtifit', and then a probabilistic diffusion model was fit using FSL's

212 'bedpostX'. Probabilistic tractography was performed between all ROIs of the RM-96

213 parcellation scheme using FSL's 'probtrackx2' function. Parameters used for tracking

214 were: 5000 seeds per voxel, 2000 steps, $0.5 \mathrm{~mm}$ step length, termination of paths that

215 loop back on themselves and rejection of paths that pass through an exclusion mask. The

216 curvature threshold was set to 0.2 .

Two SC matrices were constructed from the tractography results representing weights and lengths of connections, respectively. Weights were generated by taking the

219 number of streamlines detected between each ROI pair and dividing it by the total

220 number of streamlines that were successfully sent from the seed mask. In this way, they were corrected for the number of voxels in each seed ROI. The length of each connection,

222 defined in millimeters, was obtained by taking the median length of all connecting 
223 streamlines for each region pair. To obtain tract lengths using FSL's probtrackx2,

224 tractography was run with and without the distance correction, and dividing the results

225 gave an estimate of the lengths of each streamline. As tractography cannot provide

226 information on directionality of connections, matrices were symmetrized such that the

227 weights between areas A to B and B to A were averaged. To account for numerous false

228 positives that are known to result from probabilistic tractography (Shen, Goulas, et al.,

229 2019), any connections not present in at least $50 \%$ of the comparison subjects were set to

230 zero (Roberts, Perry, Roberts, Mitchell, \& Breakspear, 2017).

\section{Resting State fMRI}

Resting state fMRI preprocessing was done using FMRIB's FEAT toolbox. The

following steps were performed: (1) motion correction with MCFLIRT (Jenkinson,

234 Bannister, Brady, \& Smith, 2002), (2) slice timing correction, (3) spatial smoothing

$235(5 \mathrm{~mm})$, and (4) registration to anatomical volume. A nuisance regression removed signals

236 from cerebral spinal fluid, white matter, and 6 motion parameters. Global signal

237 regression was not performed. Lastly, a high-pass filter (100s) was applied.

A weighted average time series was calculated for every ROI, such that each

239 voxel was weighted according to the probability it was within a given ROI (all voxels

240 summed to one), with voxels more central in the ROI being favoured (Shen et al., 2012).

241 This approach aimed to minimize partial volume bias. A FC matrix was calculated for

242 each subject by finding the pairwise Pearson's correlation coefficient between each ROI

243 pair's weighted average time series. To characterize the dynamics of the resting

244 fluctuations, we calculated functional connectivity dynamics (FCD) matrices for each 
245 subject. The 7-minute resting state scan was split into 92 windows of 30 seconds

246 overlapping by 4 seconds. We calculated the FC matrix for each time window centred at

247 time $t$, generating a timeseries of FC matrices, $\mathrm{FC}(t)$. The FCD matrix is a $t$ by $t$ matrix

248 with element $(t 1, t 2)$ calculated as the Pearson's correlation of the upper triangle's the FC

249 matrices $\mathrm{FC}(t 1)$ and $\mathrm{FC}(t 2)$. This method of characterizing resting fluctuations with $\mathrm{FCD}$

250 has been used previously (Deco, Kringelbach, Jirsa, \& Ritter, 2017).

251 No participants were removed due to head motion during the resting state fMRI

252 scan (absolute head motion at a single time point was no greater than $3.5 \mathrm{~mm}$ and relative

253 head motion did not exceed $2.5 \mathrm{~mm}$ for any participants. A one-way ANOVA showed the

254 comparison participants $(M=0.27, S D=0.28), \mathrm{CT} / \mathrm{MRI}$ positive patients $(M=0.25$,

$255 S D=0.20)$ and CT/MRI negative patients $(M=0.23, S D=0.17)$ did not differ in mean

256 absolute head motion, $\mathrm{F}(2,77)=0.22, \mathrm{p}=0.801$. Similarly the comparison participants

257 ( $M=0.06, S D=0.03)$, CT/MRI positive $(M=0.07, S D=0.02)$, and CT/MRI negative patients

$258(M=0.10, S D=0.09)$ did not differ in mean relative head motion, $\mathrm{F}(2,77)=2.00, \mathrm{p}=0.14$.

\section{The Virtual Brain}

We simulated fMRI time series for each subject. Simulations were constrained by 261 each subject's empirical SC and fitted to their FC and FCD matrices. The process for

262 each subject may be summarized as follows: (1) SC matrices (weights and tract lengths)

263 are input to fast_tvb (https://github.com/BrainModes/fast_tvb,

264 https://hub.docker.com/r/thevirtualbrain/fast_tvb); (2) local and global parameters are

265 chosen for the model; (3) fMRI is simulated based on local and global parameters and

266 constrained by individual SC; (4) individual fMRI simulations are optimized by re- 
267 running simulations with different local and/or global parameter values that yielded the

268 best fit; (5) best-fitting local and global parameters are used for group comparisons, and

269 to account for individual variability on our neuropsychological factors. The modeling

270 process in TVB has been described more thoroughly previously (Ritter et al., 2013; Sanz

271 Leon et al., 2013) and the mean field approximations used have been validated

272 independently (Deco \& Hugues, 2012). Readers may also refer to an excellent, more

273 general review of dynamic models of large-scale brain activity (Breakspear, 2017).

\section{Dynamic Mean Field Model}

The dynamic mean field model represents each region of interest as a population

276 of excitatory and inhibitory neurons coupled by excitatory NMDA synapses and

277 inhibitory GABA synapses. The model is defined by a set of six stochastic nonlinear

278 differential equations, modified slightly from those presented by Deco et al. (2014), such

279 that the global, inter-region connections incorporated time delays (Deco, McIntosh, et al.,

280 2014; Deco, Ponce-Alvarez, et al., 2014).

$281 I_{i}^{(E)}(t)=W_{E} I_{0}+w_{+} J_{N M D A} S_{i}^{(E)}(t)+G J_{N M D A} \sum_{j} C_{i j} S_{j}^{(E)}\left(t-\frac{D_{i j}}{s}\right)-J_{i} S_{i}^{(I)}(\mathrm{t})$

$I_{i}^{(I)}(t)=W_{I} I_{0}+J_{N M D A} S_{i}^{(E)}(t)-S_{i}^{(I)}(t)$

$$
r_{i}^{(E)}(t)=\frac{a_{E} I_{i}^{(E)}(t)-b_{E}}{1-\exp \left(-d_{E}\left(a_{E} I_{i}^{(E)}(t)-b_{E}\right)\right)}
$$

$284 \quad r_{i}^{(I)}(t)=\frac{a_{I} I_{i}^{(I)}(t)-b_{I}}{1-\exp \left(-d_{I}\left(a_{I} I_{i}^{(I)}(t)-b_{I}\right)\right)}$ 


$$
\begin{aligned}
& \frac{d s_{i}^{(E)}(t)}{d t}=\frac{-S_{i}^{(E)}(t)}{\tau_{E}}+\left(1-S_{i}^{(E)}(t)\right) \gamma r_{i}^{(E)}(t)+\sigma v_{i}(t) \\
& \frac{d s_{i}^{(I)}(t)}{d t}(t)=\frac{-S_{i}^{(I)}(t)}{\tau_{I}}+r_{i}^{(I)}(t)+\sigma v_{i}(t)
\end{aligned}
$$

$$
\mathrm{I}_{\mathrm{i}}^{(\mathrm{E})} \text { represents the input current to the excitatory population of region } i \text {, while } \mathrm{I}_{\mathrm{i}}^{(\mathrm{I})}
$$

288 denotes the input current to the inhibitory population at that region. Equations 3 and 4

289 convert input current to firing rates, $r_{i}^{(E / I)}$, for the excitatory, and inhibitory populations of 290 region $i$ respectively. Finally, the firing rate is used to calculate synaptic gating $\left(\mathrm{S}_{\mathrm{i}}^{(\mathrm{E} / \mathrm{I})}\right)$ of

291 both the excitatory and inhibitory populations in equations 5 and 6 .

Input current to the excitatory population is defined by four sources: the overall

effective external current $\left(W_{E} I_{0}\right)$, recurrent excitatory currents $\left[w_{+} J_{N M D A} S_{i}^{(E)}(t)\right]$,

294 recurrent inhibitory currents $\left(J_{i} S_{i}^{(I)}(t)\right)$, and excitatory currents from other region's

295 excitatory populations $\left[G J_{N M D A} \sum_{j} C_{i j} S_{j}^{(E)}\left(t-\frac{D_{i j}}{s}\right)\right]$. Long-range (inter-region)

296 connections between regions $i$ and $j$ are constrained by the connectivity weight $C_{i j}$, where

$297 C_{i j}$ is the $(i, j)$-th entry in the $\mathrm{SC}$ weights matrix. Global coupling strength, $G$, scales the

298 long-range connectivity weights $C_{i j}$. Time delays are incorporated through the division of

299 the distance between regions $i$ and $j,\left(D_{i j}\right)$, by conduction velocity, $s$. The input current to

300 the inhibitory population is defined by: external currents $\left(W_{I} I_{0}\right)$, recurrent excitatory

301 currents $\left[J_{N M D A} S_{i}^{(E)}(t)\right]$, and recurrent inhibitory currents $\left[S_{i}^{(I)}(t)\right]$. Notably, noise is

302 added in equations 5 and 6 , where $v_{i}$ is uncorrelated standard Gaussian noise, with

303 amplitude scaled by $\sigma$. 
To maintain an average firing rate between $2-5 \mathrm{~Hz}$ feedback inhibition control

305 (FIC) was applied (Deco, Ponce-Alvarez, et al., 2014; Schirner et al., 2018). The FIC

306 algorithm iteratively adjusts the inhibitory connection weights $\left(J_{i}\right)$ at each region, so that

307 each local excitatory population maintains an average firing rate of $\sim 3 \mathrm{~Hz}$. By

308 maintaining local excitation-inhibition balance in this way, we produce simulations that

309 fit better with empirical fMRI and show more realistic firing rates (Deco, Ponce-Alvarez,

310 et al., 2014).

311 The differential equations were integrated with a step size of $0.1 \mathrm{~ms}$. After starting

312 a simulation, first the $J_{i}$ values were fitted using an automatic routine, and then 8 minutes

313 and 20 seconds of fMRI were simulated to match the duration of the empirical fMRI

314 timeseries. Eighty seconds of simulated fMRI data were removed to account for initial

315 transients. Simulated synaptic activity was fed through the Balloon-Windkessel

316 hemodynamic model producing simulated fMRI data (Friston et al., 2000). All

317 simulations were performed using an implementation of the dynamic mean field model in

318 C (Schirner et al., 2018; code available: https://github.com/BrainModes/fast tvb).

Subject-specific parameter space explorations found the best-fitting value of

321 global coupling to maximize their simulation's fit to their empirical FC and FCD. To

322 avoid overfitting we used two features to define goodness-of-fit. Our first feature was

323 maximal uncentered Pearson correlation of the upper triangle of each subject's empirical

324 and simulated FC matrices. The second was the minimal Kolmogorov-Smirnov distance

325 between the distributions formed by the upper triangle's of the simulated and empirical 
326 FCD matrices. Uncentered Pearson correlation takes into account the difference in the

327 mean values of the FC matrices (Deco, Ponce-Alvarez, et al., 2014) and fitting FCD

328 matrix distributions with Kolmogorov-Smirnov distance has been used previously to

329 effectively fit brain dynamics in network models (Deco, Kringelbach, Jirsa, \& Ritter,

330 2017). We explored 50 values of $G$ for each subject and to account for the effect of intra-

331 subject variability due to our stochastic model, we ran 20 iterations of the PSE for each

332 subject with randomized initial conditions, resulting in 1000 simulations being run for

333 every subject. Regional inhibitory connection strengths were fitted for each of these

334 simulations via the FIC tuning algorithm described in the previous section. For each

335 iteration of the PSE, we ranked the FC and FCD fits for each value of $\mathrm{G}$ and chose the

336 optimal $G$ value based on the best-combined rank. Then we chose the value of $G$ that

337 most frequently (mode) produced the best combined FC/FCD fit. We also used the

338 regional inhibitory connection weights $\left(\mathrm{J}_{\mathrm{i}}\right)$ for the best-fitting value of $G$, found by the

339 FIC algorithm. We refer to global coupling and regional inhibitory connection weights

340 (97 variables total) as the 'TVB parameters' collectively. Note that global coupling was

341 used to maximize fit with empirical fMRI, while the fitting target for regional inhibitory

342 connection strength was the average firing rate of the excitatory population at each

343 region.

\section{$344 \quad$ Partial Least Squares}

345 Partial least squares (PLS) is a multivariate statistical method that relates two sets

346 of variables by identifying linear combinations of variables in both sets that maximally

347 covary together (McIntosh \& Lobaugh, 2004; McIntosh \& Mišić, 2013). We used PLS to

348 find optimal relationships between a set of brain variables and either a study design 
349 (mean-centering PLS) or a set of behavioural variables (behavioural PLS). In PLS,

350 singular value decomposition (SVD) is used to find orthogonal latent variables that

351 explain the maximal amount of covariance between brain variables and design or

352 behaviour variables. For each latent variable brain saliences are calculated for each brain

353 region that indicate the degree to which each region contributes to the relationship

354 between brain and design/behaviour expressed by the latent variable. In mean-centering

355 PLS, design saliences indicate the group, condition, or group $x$ condition profiles that best

356 describe the relationship between the set of brain and design variables. In behavioural

357 PLS, behaviour saliences indicate the profile of behaviour variables that best characterize

358 the relationship between brain and behaviour variables. Brain scores are calculated for

359 each subject and latent variable by multiplying the matrix of brain variables by the brain

360 saliences. Brain score indicates the degree to which each participant contributes to each

361 latent variable. Lastly, singular values are the covariance between brain and

362 behaviour/grouping variables. They can also be evaluated as percentage of the total

363 covariance between measures that each latent variable accounts for.

In our study, we used mean centering PLS to determine the relationship between

365 several brain measures and group status. The brain measures considered were a

366 vectorized version of the upper triangle of the $\mathrm{SC} / \mathrm{FC}$ matrices, FA values from all voxels

367 within our white matter mask and TVB parameters (global coupling and regional

368 inhibitory connection strength values). For each brain measure, an omnibus PLS was

369 performed that found the optimal contrasts in brain measures between group membership.

370 We also used behavioural PLS to determine the relationships between the TVB 
371 parameters (global coupling and regional inhibitory connection strength) and factor

372 scores from a set of patient outcome variables.

Permutation testing was used to determine the significance of each latent variable.

374 Rows of the data matrix were randomly reordered and the singular value recalculated.

375 This was done 1000 times, creating a distribution of singular values. Then a $p$-value for

376 the original singular value was calculated by taking the proportion of singular values

377 from the sampling distribution that were larger than the original singular value. The $p$ -

378 value can be thought of the probability of obtaining a singular value of this size under the

379 null hypothesis that there is no association between brain measure and design/behaviour.

380 Bootstrapping was used to estimate the reliability of each brain salience.

381 Participants were randomly resampled 1000 times with replacement, while respecting

382 group membership. The resampled matrices were used to recalculate the singular vector

383 decomposition, producing a sampling distribution for the weights in the singular vectors.

384 Standard error was calculated from this sampling distribution, reflecting the stability of

385 the weight regardless of which participants are included in the analysis. Then a bootstrap

386 ratio (BSR) was calculated for each brain salience (voxel, brain region, connection) by

387 dividing the brain salience by its bootstrap-estimate standard error. BSR is akin to z-score

388 (|2.0| corresponds to approximately a $95 \%$ confidence interval) but is interpreted in terms

389 of reliability of the parameter rather than null hypothesis testing. Confidence intervals

390 were calculated around design/behavior salience using the percentiles derived from the

391 sampling distribution. 


\section{Outcome Measures}

Our PCA of background and outcome variables identified two factors: (1) TBI

Symptoms, (2) Age and Cognition (Table 5), consistent with a previously reported PCA of neuropsychological outcome variables in mTBI patients (Levin et al., 2013). The

397 CT/MRI subgroups did not significantly differ on the TBI Symptoms factor $(t(34)=0.73$, $398 p=0.47)$, or the Age and Cognition factor $(t(34)=0.30, p=0.76)$. We also tested for group 399 differences on the individual variables contributing to both factors. We found the $400 \mathrm{CT} / \mathrm{MRI}$ positive patients were significantly older than the CT/MRI negative patients $401(t(42)=-2.5, p<0.01)$ and comparison participants $(t(48)=4.3, p=<0.01)$. The CT/MRI 402 negative patients were also significantly older than the comparison participants $403(t(64)=2.2, p=0.03)$. A chi-square test of independence indicated that the CT/MRI 404 positive patients experienced a greater loss of consciousness than the CT/MRI negative 405 patients $\left(X(1)^{2}=8.6, \mathrm{p}=0.01\right)$. There were no other significant differences between groups $406(p>0.05$, see Table 1$)$.

407 Empirical brain differences between patient subgroups and comparison participants

\section{Structural connectivity}

A group comparison PLS assessed potential differences in structural connectivity

410 weights between the CT/MRI patient subgroups and comparison participants. The first

411 latent variable $(p<0.0001,67 \%$ covariance, singular value $=0.19)$ distinguished the

$412 \mathrm{CT} / \mathrm{MRI}$ positive patients from comparison participants (Figure 2A-C), indicating 
$413 \mathrm{CT} / \mathrm{MRI}$ positive patients had lower SC weights relative to the comparison participants in

414 many voxels, indicating a global effect. The second latent variable $(p=0.016,33 \%$

415 covariance, singular value $=0.13$ ) distinguished $\mathrm{CT} / \mathrm{MRI}$ negative patients from

416 comparison participants (Figure 2D-F) showing the CT/MRI negative patients also had

417 primarily lower SC than comparison participants especially in connections involving

418 occipital regions. These analyses were repeated with age regressed from the SC values

419 and the results did not change appreciably.

\section{Functional connectivity and functional connectivity dynamics}

A group comparison PLS comparing functional connectivity across the CT/MRI

patient subgroups and comparison participants did not find any significant differences

$(p=0.58,61.7 \%$ covariance, singular value $=3.1)$. A one-way ANOVA did not

424 discriminate the patient groups or comparison subjects on the variance of their FCD

425 matrices, $F(2,77)=0.1, p=0.91$. These analyses were repeated with age regressed from the

426 FC values and the results did not change appreciably.

\section{Fractional anisotropy}

A group comparison PLS compared fractional anisotropy across the whole-brain

429 white matter skeleton (Figure 3$)$. The first latent variable ( $p=0.01,65.1 \%$ covariance,

430 singular value $=8.2$ ) showed CT/MRI positive patients had lower FA than the comparison

431 group, especially in the left cingulum and anterior corona radiata. The second latent

432 variable indicated CT/MRI negative patients had primarily lower FA than comparison

433 participants, though it did not reach significance $(p=0.09,34.9 \%$ covariance, singular 
434 value $=6.0$ ). These analyses were repeated with age regressed from the FA values and the

435 results did not change meaningfully.

\section{TVB Model Fitting}

437 All patients and comparison participants showed good fits between empirical and 438 simulated resting state fMRI, as assessed by high uncentered Pearson's correlation of FC 439 and low Kolmogorov-Smirnov distance between FCD matrices. The simulated FC and 440 FCD matrices of all participants were inspected visually to ensure they appeared as 441 reasonable FC/FCD matrices qualitatively. We also screened for outliers by converting 442 the FC and FCD fits to z-score, finding no participants with a z-scored FC fit lower than 4433 (minimum $=-2.4$ ) and no participants with a z-scored FCD fit higher than 3 (maximum $444=2.6$ ). There were no significant differences between the three groups on the fitting 445 metrics (Table 5). 
446 The parameter search space showed steady improvements in fit (increased FC

447 correlation and decreased FCD Kolmogorov-Smirnov distance) as global coupling was

448 increased until a best-fitting global coupling value was reached (Figure 4). After this

449 point, the model becomes multistable and eventually the FIC tuning algorithm is no

450 longer able to maintain biologically realistic firing rates of $\sim 3 \mathrm{~Hz}$. Across multiple

451 iterations our model showed acceptable consistency in choosing the optimal value of

452 global coupling across iterations (Table 6, Figure 4).

\section{TVB model group differences}

\section{Combined TBI patients versus comparison subjects}

A group comparison PLS compared the TVB parameters (global coupling and

456 regional inhibitory connection strengths) of the comparison participants ( $\mathrm{n}=36)$ and the

457 combined CT/RMI positive and negative patient groups $(\mathrm{n}=44)$. A significant latent

458 variable ( $p=0.026$, singular value $=0.30$; Figure 5 ) distinguished the groups, showing the

459 TBI patients had primarily higher inhibitory connection strengths, especially in bilateral

460 subcortical regions. Regions that were reliably contributing (bootstrap ratio $>2$ ) to the

461 relationship included the bilateral pallidum, a right thalamic ROI with major temporal

462 connections and right thalamic ROI with major frontal connections, right superior

463 temporal cortex, left inferior cortex, and left putamen. The results were not significantly

464 affected when age was regressed from the variables and the analysis repeated. 

regional inhibitory connection strengths) of the CT/MRI positive patients $(\mathrm{n}=14)$, CT/MRI negative patients $(n=30)$, and comparison participants $(n=36)$. The first significant latent variable $(p=0.03,70.9 \%$ covariance, singular value $=0.43$; Figure $6 \mathrm{~A}-\mathrm{C}$ ) distinguished the CT/MRI positive patients from comparison participants, showing that they had primarily lower cortical and higher subcortical inhibitory connection strengths relative to the comparison subjects. Regions reliably lower in CT/MRI positive patients compared to comparison participants (bootstrap ratio $<-2$ ) included the left dorsomedial prefrontal cortex, left medial prefrontal cortex, left prefrontal polar cortex, left anterior insula, right centrolateral prefrontal cortex, right medial prefrontal cortex, and right orbitoinferior prefrontal cortex. On the other hand, the left/right pallidum were reliably higher in CT/MRI positive patients relative to comparison participants. The dot product of the brain salience vector from the first latent variable (Figure 6) and the brain salience vector from the group comparison PLS comparing the combined patients from comparison subjects (Figure 5) was high $(\mathrm{r}=0.77)$ indicating the $\mathrm{CT} / \mathrm{MRI}$ positive patients were likely largely responsible for driving the group difference. These analyses were repeated with age regressed from the TVB parameters and the results did not change meaningfully.

\section{TVB-behaviour relationships}

A behavioural PLS analysis compared the correlations between the TVB 
487 ( $\mathrm{N}=36)$. The analysis showed higher scores on the TBI Symptoms (indicating more

488 symptoms) and Age and Cognition (indicating older age and poorer cognitive

489 performance) factors were related to primarily lower regional inhibitory connection

490 strength ( $p=0.02,74 \%$ covariance, singular value $=2.4$; Figure 7$)$. Regions reliably

491 contributing to the latent variable (bootstrap ratios $<-2$ ) included the left/right gustatory

492 cortex, the left caudate nucleus, secondary auditory cortex, primary visual cortex,

493 hippocampus, orbitomedial prefrontal cortex, anterior insula, and temporal polar cortex,

494 as well as the right frontal eye field, ventral temporal cortex, anterior visual area (ventral

495 part), ventrolateral premotor cortex, gustatory cortex, and inferior temporal cortex.

Within group PLS models were calculated to determine the contributions of the

497 CT/MRI positive and negative groups to the omnibus test shown in Figure 8. Both PLS

498 analyses compared TBI Symptoms and Age and Cognition factor scores to the TVB

499 parameters (global coupling and regional inhibitory connection strength) using either the

$500 \mathrm{CT} / \mathrm{MRI}$ positive or negative patients. The within CT/MRI negative behavioural PLS

501 produced a single significant latent variable $(\mathrm{n}=25 ; p=0.005,73.1 \%$ covariance, singular

502 value=3.0; Figure 8A-C) indicating higher TBI Symptoms and Age and Cognition scores

503 were related to lower inhibitory connection strength. Regions reliably contributing to the

504 latent variable (bootstrap ratios $<-2$ ) included the right temporal polar cortex,

505 orbitomedial prefrontal cortex, parahippocampal cortex, anterior visual area, ventral

506 temporal cortex, and frontal eye field, as well as the left anterior insula, orbitomedial

507 prefrontal cortex, hippocampus, frontal eye field, dorsolateral prefrontal cortex, and

508 caudate nucleus. The left subgenual cingulate gyrus showed the inverse association with

509 the factor scores compared to the other regions (bootstrap ratio $>2$ ). The same analysis 
510 within the CT/MRI positive group did not produce a significant latent variable $(\mathrm{n}=11$,

$511 p=0.11,67.6 \%$ covariance, singular value $=3.7$; Figure $8 \mathrm{D}-\mathrm{F})$. We calculated the dot

512 product of the brain salience vectors from the combined group PLS model (Figure 7) and

513 the two within group analyses (Figure 8) to determine which subgroup was responsible

514 for driving the effect observed when groups were combined. The dot product between the

515 combined model and the CT/MRI negative model was very high $(\mathrm{r}=0.90)$. The same dot

516 product was significantly lower $(\mathrm{r}=-0.43)$ between the combined model and the CT/MRI

517 positive group ( $z=4.68, p<0.0001)$, suggesting the CT/MRI negative group was primarily

518 responsible for the observed associations between TBI Symptoms and Age and Cognition

519 factor scores and regional inhibitory connection strengths.

\section{Discussion}

\section{Overview}

Concussion outcomes are variable and difficult to predict. Few studies have

523 looked longitudinally at patients with well-characterized acute and chronic phase

524 assessments and fewer still have combined multimodal imaging with a computational

525 approach. Here we combined the strengths of a well-characterized sample with

526 multimodal imaging and computational approaches to reveal relationships between local

527 neural dynamics and chronic patient outcomes. We found that the CT/MRI positive TBI

528 patients showed lower cortical, but higher subcortical inhibitory connection strengths

529 relative to the comparison participants. We did not distinguish CT/MRI negative patients

530 from the comparison participants based on their TVB parameters, however, lower

531 regional inhibitory connection strengths were related to higher scores on the TBI 
532 Symptoms and Age and Cognition factors assessed six months post-injury in these

533 patients. Together, our results show proof of concept that local brain dynamics modeled

534 in TVB are sensitive to semi-acute TBI pathology and predictive of chronic outcomes.

\section{Group Comparisons}

The CT/MRI positive patients displayed higher subcortical, but lower cortical

537 inhibitory connection strengths relative to the comparison participants. We speculate that

538 lower inhibitory connection strength may reflect excitotoxicity or inhibitory

539 dysregulation, which are known to play a role in secondary injury after TBI (Van Horn,

540 Bhattrai, \& Irimia, 2017; Yi \& Hazell, 2006). Indeed, a study of tumour patients (Aerts et

541 al., 2018) that used a similar model to our own demonstrated reduced inhibitory

542 connection strength in tumour regions relative to comparison participants, which they

543 posited to reflect the role of excitotoxicity in the pathogenesis of glaucoma (Casson,

544 2006). Aerts et al. (2018) also observed increased inhibitory connection strengths in the

545 non-tumour regions of their patients compared to controls, paralleling the increased

546 inhibitory connection strengths we observed in the subcortical regions of the CT/MRI

547 positive patients compared to comparison participants. Higher subcortical inhibitory

548 connection strengths may reflect a distal effect of small cortical lesions, or serve a

549 protective function, as CT/MRI positive patients did not score more highly than CT/MRI

550 negative patients on the TBI Symptoms factor. Future studies using neuroimaging with

551 finer spatial resolution could quantitatively assess the sensitivity of our model's regional

552 inhibitory connection strength parameter to the cellular processes underlying changes to

553 excitation/inhibition caused by TBI. 
The CT/MRI negative patients were not significantly differentiated from the

555 comparison participants by their TVB parameters. This was unexpected as a study of

556 mTBI patients experiencing active PCS symptoms (who were screened for focal lesions)

557 found decreased inhibitory connection strengths relative to comparison participants

558 (Good et al., in preparation). Notably, the present work investigates TBI patients scanned

559 1-2 weeks post-injury, while the other study considers patients in the chronic phase post-

560 injury, which may contribute to the discrepant findings. It is also possible that other

561 factors obfuscated a potential group difference between CT/MRI negative patients and

562 comparison participants. For example, history of substance abuse and other

563 neuropsychiatric disorders, such as depression, were not exclusion criteria in the present

564 study despite exerting effects via excitotoxicity (Miller, Maletic, \& Raison, 2009; Walker

565 \& Dantzer, 2014) similarly to mTBI. While this feature of our data increases our

566 finding's generalizability, it may also have limited our sensitivity to pathophysiology

567 caused by TBI.

Our empirical SC and FA findings suggested the CT/MRI positive and negative

569 patients were characterized by more disconnected structural connectomes than the

570 comparison participants. These results are in line with a previous study of the white

571 matter integrity of an overlapping patient sample (Yuh et al., 2014). In addition, previous

572 studies of humans (Imms et al., 2019; Kuceyeski, Jamison, Owen, Raj, \& Mukherjee,

573 2019) and animals (Meningher, Bernstein-Eliav, Rubovitch, Pick, \& Tavor, 2020) have

574 found the SC of TBI patients are more highly segregated than controls. Modeling studies

575 suggest structural disconnection in TBI patients produces reduced metastability, linked to

576 excitation-inhibition imbalance (Hellyer, Scott, Shanahan, Sharp, \& Leech, 2015). 
577 Similarly, the CT/MRI positive patients demonstrated structural disconnection as well as

578 altered local inhibitory dynamics compared to comparison participants.

579 When interpreting our findings, it should be considered that approximately one 580 third of our TBI sample (14 of 44 patients) showed signs of acute traumatic intracranial 581 lesions on CT or MRI scan upon presentation in the emergency department. This 582 classification of CT/MRI positive versus CT/MRI negative may be contrasted with 583 previous work considering 'complicated' mTBI solely on the basis of a positive acute 584 phase CT scan (Iverson et al., 2012; Voormolen et al., 2019). Many of our CT/MRI 585 positive patients were classified on the basis of very subtle MRI lesions at 3T, such as 586 one or two subtle isolated foci of hemorrhagic axonal injury. These patients would likely 587 be classified as uncomplicated if we had used CT alone. In fact, this nuance is supported 588 by early work that found dw-MRI evidence of white matter damage in patients classified 589 as uncomplicated by CT alone (Arfanakis et al., 2002; Bazarian et al., 2007; Chu et al., 590 2010).

\section{TVB-Behaviour Relationships} Lower inhibitory connection strengths were associated with higher scores on the

593 TBI Symptoms and Age and Cognition factors in the CT/MRI negative group. The effect 594 included many regions including cortical and subcortical structures, and indicated that 595 lower inhibitory connection strengths were related to more severe TBI outcomes, older 596 age, less education, and lower cognitive performance. This effect is aligned with other 597 large-scale modeling work that showed structural disconnection associated with 598 decreased metastability, indicating excitation-inhibition imbalance, to be related to poorer 
599 cognitive performance in TBI patients (Hellyer et al., 2015). Empirical studies using

600 magnetic resonance spectroscopy have also found elevated concentrations of the

601 neuromodulatory factor glutamate during the semi-acute phase were predictive of poorer

602 chronic outcomes (Eisele et al., 2020; Shutter et al., 2004).

In the CT/MRI positive patients we did not observe a correlation between

604 inhibitory connection strengths and the TBI Symptoms factor as expected. We suspect

605 this was due to a lack of statistical power $(n=11)$ in the within group behavioural PLS

606 (Figure 8D-F). Forthcoming large-scale longitudinal and multi-site data acquisitions

607 (such as full Track-TBI LONG [(https://tracktbi.ucsf.edu/] sample) will be better

608 equipped to establish robust correlations between parameters from brain network models

609 and chronic TBI outcome.

610 Limitations and Conclusions

611 We note our study is limited by its modest sample size and lack of longitudinal

612 imaging data to track recovery. Our exploratory findings will need to be confirmed in

613 larger independent samples. Specifically, future studies should test whether lower

614 inhibitory connection strength is indeed predictive of development of persistent post-

615 concussion symptoms. Additionally, we acknowledge the lack of racial diversity (79\%

616 white) and the gender imbalance ( $65 \%$ male) of our patients. Our sample, obtained by an

617 emergency room convenience sample, will limit the generalizability of our findings to

618 more mild concussions that do not require hospitalization. Our choice of a relatively

619 coarse (96 ROI) parcellation scheme may have also limited sensitivity. Future work

620 should explore the effect of multiple parcellation schemes on modeling results. Similarly, 
621 future work with larger sample sizes may allow for subtyping moderate to severe TBI

622 patients by lesion type and location, which would allow for greater power in detecting

623 changes to local network dynamics. Regarding our network model, we acknowledge a

624 mechanistic interpretation may not be possible as multiple processes at the micro-level

625 may contribute to similar observations at the macro-level. For example, local excitatory

626 connection strengths may produce similar dynamics as those observed through variations

627 to recurrent inhibitory connection strengths. We did not vary local excitatory connection

628 strengths in our parameter space exploration to ensure the model remained identifiable.

629 In conclusion, we used large-scale brain modeling to detect differences in local

630 inhibitory connection strengths between CT/MRI positive TBI patients in the semi-acute

631 phase and comparison participants. We did not distinguish semi-acute CT/MRI negative

632 patients from the comparison participants based on their inhibitory connection strengths,

633 however, lower inhibitory connection strengths were associated with more severe clinical

634 outcomes, older age and poorer cognitive performance at a 6-month follow-up in these

635 patients. The result suggests large-scale connectome based models may be sensitive to

636 pathophysiological changes in semi-acute phase TBI patients and predictive of their

637 chronic outcomes. 
639 Aerts, H., Schirner, M., Jeurissen, B., Van Roost, D., Achten, E., Ritter, P., \& Marinazzo, 640 D. (2018). Modeling Brain Dynamics in Brain Tumor Patients Using the Virtual 641 Brain. Eneuro, 5(3), ENEURO.0083-18.2018. https://doi.org/10.1523/eneuro.0083$642 \quad 18.2018$

643 Arfanakis, K., Haughton, V. M., Carew, J. D., Rogers, B. P., Dempsey, R. J., \& 644 Meyerand, M. E. (2002). Diffusion tensor MR imaging in diffuse axonal injury. AJNR. American Journal of Neuroradiology, 23(5), 794-802.

Avants, B. B., Tustison, N. J., Song, G., Cook, P. A., Klein, A., \& Gee, C. (2011). A Reproducuble Evaluation of ANTs Similarity Metric Performance in Brain Image Registration. NeuroImage, 54(3), 2033-2044. https://doi.org/10.1016/j.neuroimage.2010.09.025.A

Bazarian, J. J., Zhong, J., Blyth, B., Zhu, T., Kavcic, V., \& Peterson, D. (2007). Diffusion

Bezgin, G., Solodkin, A., Bakker, R., Ritter, P., \& Mcintosh, A. R. (2017). Mapping Complementary Features of Cross-Species Structural Connectivity to Construct Realistic “Virtual Brains .” Human Brain Mapping, 38, 2080-2093. https://doi.org/10.1002/hbm.23506

Breakspear, M. (2017). Dynamic models of large-scale brain activity. Nature Neuroscience, 20(3), 340-352. https://doi.org/10.1038/nn.4497

660 Casson, R. J. (2006). Possible role of excitotoxicity in the pathogenesis of glaucoma. Clinical and Experimental Ophthalmology, 34(1), 54-63. https://doi.org/10.1111/j.1442-9071.2006.01146.x

663 Chu, Z., Wilde, E. A., Hunter, J. V., McCauley, S. R., Bigler, E. D., Troyanskaya, M., ... 
664

665

666

667

668

669

670

671

672

673

674

675

676

677

678

679

680

681

682

683

684

685

686

687

688

689

690

Levin, H. S. (2010). Voxel-based analysis of diffusion tensor imaging in mild traumatic brain injury in adolescents. American Journal of Neuroradiology, 31(2), 340-346. https://doi.org/10.3174/ajnr.A1806

Dabek, F. J., \& Caban, J. J. (2016). A K-Reversible Approach to Model Clinical Trajectories. AMIA ... Annual Symposium Proceedings. AMIA Symposium, 2016, $460-469$.

Deco, G., McIntosh, A. R., Shen, K., Hutchison, R. M., Menon, R. S., Everling, S., ... Jirsa, V. K. (2014). Identification of Optimal Structural Connectivity Using Functional Connectivity and Neural Modeling. Journal of Neuroscience, 34(23), 7910-7916. https://doi.org/10.1523/JNEUROSCI.4423-13.2014

Deco, G., Ponce-Alvarez, A., Hagmann, P., Romani, G. ., Mantini, D., \& Corbetta, M. (2014). How local excitation-inhibition ratio impacts the whole brain dynamics. The Journal of Neuroscience, 34(23), 7886-7898. https://doi.org/10.1523/JNEUROSCI.5068-13.2014

Deco, Gustavo, \& Hugues, E. (2012). Neural network mechanisms underlying stimulus driven variability reduction. PLoS Computational Biology, 8(3). https://doi.org/10.1371/journal.pcbi.1002395

Deco, Gustavo, Kringelbach, M. L., Jirsa, V. K., \& Ritter, P. (2017). The dynamics of resting fluctuations in the brain: Metastability and its dynamical cortical core. Scientific Reports, 7(1), 1-14. https://doi.org/10.1038/s41598-017-03073-5

Deco, Gustavo, Tononi, G., Boly, M., \& Kringelbach, M. L. (2015). Rethinking segregation and integration: contributions of whole-brain modelling. Nature Reviews Neuroscience, 16(7), 430-439. https://doi.org/10.1038/nrn3963

Derogatis, L. R. (1983). The Brief Symptom Inventory: An Introductory Report. Psychological Medicine, 13(3), 595-605. https://doi.org/10.1017/S0033291700048017

Diener, E. R., Emmons, R., RJ, L., \& Griffin, S. (1985). The Satisfaction With Life Scale. 
691

692

693

694

695

696

697

698

699

700

701

702

703

704

705

706

707

708

709

710

711

712

713

714

715

716

717

Journal of Personality Assessment, 49(1), 71-75.

Douglas, D. B., Iv, M., Douglas, P. K., Anderson, A., Vos, S. B., Bammer, R., ... Wintermark, M. (2015). Diffusion tensor imaging of TBI: Potentials and challenges. Topics in Magnetic Resonance Imaging, 24(5), 241-251. https://doi.org/10.1097/RMR.0000000000000062

Eisele, A., Hill-Strathy, M. J., Michels, L., \& Rauen, K. (2020). Magnetic Resonance Spectroscopy following Mild Traumatic Brain Injury: A Systematic Review and Meta-Analysis on the Potential to Detect Posttraumatic Neurodegeneration. Neurodegenerative Diseases. https://doi.org/10.1159/000508098

Falcon, M. I., Riley, J. D., Jirsa, V., McIntosh, A. R., Elinor Chen, E., \& Solodkin, A. (2016). Functional Mechanisms of Recovery after Chronic Stroke: Modeling with the Virtual Brain. ENeuro, 3(2), ENEURO.0158-15.2016. https://doi.org/10.1523/ENEURO.0158-15.2016

Filley, C. M., \& Kelly, J. P. (2018). White Matter and Cognition in Traumatic Brain Injury. Journal of Alzheimer's Disease : JAD, 65(2), 345-362. https://doi.org/10.3233/JAD-180287

Friston, K. J., Mechelli, A., Turner, R., \& Price, C. J. (2000). Nonlinear Responses in fMRI: The Balloon Model, Volterra Kernels, and Other Hemodynamics. NeuroImage, 12(4), 466-477. https://doi.org/10.1006/nimg.2000.0630

Giza, C. C., \& Hovda, D. a. (2015). The New Neurometabolic cascade of Concussion. Neurosurgery, 75(0 4). https://doi.org/10.1227/NEU.0000000000000505.The

Hayes, J. P., Bigler, E. D., \& Verfaellie, M. (2016). Traumatic brain injury as a disorder of brain connectivity. Journal of the International Neuropsychological Society, 22(2), 120-137. https://doi.org/10.1017/S1355617715000740

Hellyer, P. J., Scott, G., Shanahan, M., Sharp, D. J., \& Leech, R. (2015). Cognitive Flexibility through Metastable Neural Dynamics Is Disrupted by Damage to the 
Structural Connectome. The Journal of Neuroscience : The Official Journal of the Society for Neuroscience, 35(24), 9050-9063. https://doi.org/10.1523/JNEUROSCI.4648-14.2015

Hou, W., Sours Rhodes, C., Jiang, L., Roys, S., Zhuo, J., Jaja, J., \& Gullapalli, R. P. (2019). Dynamic Functional Network Analysis in Mild Traumatic Brain Injury. Brain Connectivity, 9(6), 475-487. https://doi.org/10.1089/brain.2018.0629

Imms, P., Clemente, A., Cook, M., D’Souza, W., Wilson, P. H., Jones, D. K., \& Caeyenberghs, K. (2019). The structural connectome in traumatic brain injury: A meta-analysis of graph metrics. Neuroscience and Biobehavioral Reviews, 99(November 2018), 128-137. https://doi.org/10.1016/j.neubiorev.2019.01.002

Iverson, G. L., Lange, R. T., Wäljas, M., Liimatainen, S., Dastidar, P., Hartikainen, K. M., ... Öhman, J. (2012). Outcome from Complicated versus Uncomplicated Mild Traumatic Brain Injury. Rehabilitation Research and Practice, 2012, 1-7. https://doi.org/10.1155/2012/415740

Jenkinson, M., Bannister, P., Brady, M., \& Smith, S. (2002). Improved optimization for the robust and accurate linear registration and motion correction of brain images. NeuroImage, 17(2), 825-841. https://doi.org/10.1016/S1053-8119(02)91132-8

Jirsa, V. K., Proix, T., Perdikis, D., Woodman, M. M., Wang, H., Bernard, C., ... Chauvel, P. (2017). The Virtual Epileptic Patient: Individualized whole-brain models of epilepsy spread. NeuroImage, 145, 377-388. https://doi.org/10.1016/j.neuroimage.2016.04.049

Jirsa, V. K., Sporns, O., Breakspear, M., Deco, G., \& Mcintosh, A. R. (2010). Towards the virtual brain : network modeling of the intact and the damaged brain. Archives Italiennes de Biologie, 148, 189-205.

Kenzie, E. S., Parks, E. L., Bigler, E. D., Wright, D. W., Lim, M. M., Chesnutt, J. C., ... Wakeland, W. (2018). The dynamics of concussion: Mapping pathophysiology, persistence, and recovery with causal-loop diagramming. Frontiers in Neurology, 
9(APR), 1-16. https://doi.org/10.3389/fneur.2018.00203

746 Kotter, R., \& Wanke, E. (2005). Mapping brains without coordinates. Philosophical

747

748

749

750

751

752

753

754

755

756

757

758

Transactions of the Royal Society of London. Series B, Biological Sciences, 360, 751-766. https://doi.org/10.1098/rstb.2005.1625

Kötter, R., \& Wanke, E. (2005). Mapping brains without coordinates. Philosophical Transactions of the Royal Society of London. Series B, Biological Sciences, 360(1456), 751-766. https://doi.org/10.1098/rstb.2005.1625

Krainin BM, Forsten RD, Kotwal RS, Lutz RH, Guskiewicz KM. (2011). Mild traumatic brain injury literature review and proposed changes to classification. Journal of Special Operations Medicine: A Peer Reviewed Journal for SOF Medical Professionals, 11(3), 38-47.

Kuceyeski, A. F., Jamison, K. W., Owen, J. P., Raj, A., \& Mukherjee, P. (2019). Longitudinal increases in structural connectome segregation and functional connectome integration are associated with better recovery after mild TBI. Human Brain Mapping, 40(15), 4441-4456. https://doi.org/10.1002/hbm.24713

Levin, H. S., Li, X., McCauley, S. R., Hanten, G., Wilde, E. A., \& Swank, P. (2013). Neuropsychological outcome of mTBI: A principal component analysis approach. Journal of Neurotrauma, 30(8), 625-632. https://doi.org/10.1089/neu.2012.2627

Madhavan, R., Joel, S. E., Mullick, R., Cogsil, T., Niogi, S. N., Tsiouris, A. J., ... Shetty, T. (2019). Longitudinal Resting State Functional Connectivity Predicts Clinical Outcome in Mild Traumatic Brain Injury. Journal of Neurotrauma, 36(5), 650-660. https://doi.org/10.1089/neu.2018.5739

Mayer, A. R., Ling, J. M., Allen, E. A., Klimaj, S. D., Yeo, R. A., \& Hanlon, F. M. (2014). Static and Dynamic Intrinsic Connectivity following Mild Traumatic Brain Injury. Journal of Neurotrauma, 32(14), 1046-1055. https://doi.org/10.1089/neu.2014.3542

McIntosh, A R, \& Lobaugh, N. J. (2004). Partial least squares analysis of neuroimaging 
data: Applications and advances. NeuroImage, 23(SUPPL. 1), 250-263.

https://doi.org/10.1016/j.neuroimage.2004.07.020

McIntosh, Anthony Randal, \& Mišić, B. (2013). Multivariate statistical analyses for neuroimaging data. Annual Review of Psychology, 64, 499-525. https://doi.org/10.1146/annurev-psych-113011-143804

McMahon, P., Hricik, A., Yue, J. K., Puccio, A. M., Inoue, T., Lingsma, H. F., ... Vassar, M. J. (2014). Symptomatology and functional outcome in mild traumatic brain injury: Results from the prospective TRACK-TBI study. Journal of Neurotrauma, 31(1), 26-33. https://doi.org/10.1089/neu.2013.2984

Meningher, I., Bernstein-Eliav, M., Rubovitch, V., Pick, C. G., \& Tavor, I. (2020). Alterations in Network Connectivity after Traumatic Brain Injury in Mice. Journal of Neurotrauma, 2179, 2169-2179. https://doi.org/10.1089/neu.2020.7063

Miller, A. H., Maletic, V., \& Raison, C. L. (2009). Inflammation and Its Discontents: The Role of Cytokines in the Pathophysiology of Major Depression. Biological Psychiatry, 65(9), 732-741. https://doi.org/10.1016/j.biopsych.2008.11.029

Niogi, S. N., \& Mukherjee, P. (2010). Diffusion tensor imaging of mild traumatic brain injury. Journal of Head Trauma Rehabilitation, 25(4), 241-255. https://doi.org/10.1097/HTR.0b013e3181e52c2a

Palacios, E. M., Owen, J. P., Yuh, E. L., Wang, M. B., Vassar, M. J., Ferguson, A. R., ... Mukherjee, P. (2020). The evolution of white matter microstructural changes after mild traumatic brain injury: A longitudinal DTI and NODDI study. Science Advances, 6(32), eaaz6892. https://doi.org/10.1126/sciadv.aaz6892

Palacios, Eva M., Yuh, E. L., Chang, Y. S., Yue, J. K., Schnyer, D. M., Okonkwo, D. O., ... Mukherjee, P. (2017). Resting-state functional connectivity alterations associated with six-month outcomes in mild traumatic brain injury. Journal of Neurotrauma, 34(8), 1546-1557. https://doi.org/10.1089/neu.2016.4752

Ritter, P., Schirner, M., McIntosh, A. R., \& Jirsa, V. K. (2013). The virtual brain 
integrates computational modeling and multimodal neuroimaging. Brain Connectivity, 3(2), 121-145. https://doi.org/10.1089/brain.2012.0120

Roberts, J. A., Perry, A., Roberts, G., Mitchell, P. B., \& Breakspear, M. (2017). Consistency-based thresholding of the human connectome. NeuroImage, 145(March 2016), 118-129. https://doi.org/10.1016/j.neuroimage.2016.09.053

Roy, D., Sigala, R., Breakspear, M., McIntosh, A. R., Jirsa, V. K., Deco, G., \& Ritter, P. (2014). Using the Virtual Brain to Reveal the Role of Oscillations and Plasticity in Shaping Brain's Dynamical Landscape. Brain Connectivity, 4(10), 791-811. https://doi.org/10.1089/brain.2014.0252

Sanz Leon, P., Knock, S. a, Woodman, M. M., Domide, L., Mersmann, J., McIntosh, A. R., \& Jirsa, V. (2013). The Virtual Brain: a simulator of primate brain network dynamics. Frontiers in Neuroinformatics, 7, 1-23. https://doi.org/10.3389/fninf.2013.00010

Schirner, M., Mcintosh, A. R., Jirsa, V., \& Deco, G. (2018). Inferring multi-scale neural mechanisms with brain network modelling. ELife, 1-30.

Schirner, M., Rothmeier, S., Jirsa, V. K., McIntosh, A. R., \& Ritter, P. (2015). An automated pipeline for constructing personalised virtual brains from multimodal neuroimaging data. NeuroImage, 117, 343-357. https://doi.org/10.1016/j.neuroimage.2015.03.055

Sharp, D. J., Scott, G., \& Leech, R. (2014). Network dysfunction after traumatic brain injury. Nature Reviews Neurology, 10(3), 156-166. https://doi.org/10.1038/nrneurol.2014.15

Shen, K., Bezgin, G., Hutchison, R. M., Gati, J. S., Menon, R. S., Everling, S., \& McIntosh, A. R. (2012). Information processing architecture of functionally defined clusters in the macaque cortex. The Journal of Neuroscience, 32(48), 17465-17476. https://doi.org/10.1523/JNEUROSCI.2709-12.2012

Shen, K., Bezgin, G., Schirner, M., Ritter, P., Everling, S., \& McIntosh, A. R. (2019). A 
826

827

828

829

830

831

832

833

834

835

836

837

838

839

840

841

842

843

844

845

846

847

848

849

850

851

852

macaque connectome for large-scale network simulations in TheVirtualBrain.

Scientific Data, 6(1), 1-12. https://doi.org/10.1038/s41597-019-0129-z

Shen, K., Goulas, A., Grayson, D., Eusebio, J., Gati, J. S., \& Ravi, S. (2019). Exploring the limits of network topology estimation using diffusion-based tractography and tracer studies in the macaque cortex. Neuoroimage (in Press), 1-37. https://doi.org/https://doi.org/10.1016/j.neuroimage.2019.02.018

Shenton, M. E., Hamoda, H. M., Schneiderman, J. S., Bouix, S., Pasternak, O., Rathi, Y., ... Zafonte, R. (2012). A review of magnetic resonance imaging and diffusion tensor imaging findings in mild traumatic brain injury. Brain Imaging and Behavior, 6(2), 137-192. https://doi.org/10.1007/s11682-012-9156-5

Shutter, L., Tong, K. A., \& Holshouser, B. A. (2004). Proton MRS in acute traumatic brain injury: Role for glutamate/glutamine and choline for outcome prediction. Journal of Neurotrauma, 21(12), 1693-1705. https://doi.org/10.1089/neu.2004.21.1693

Si, B., Dumkrieger, G., Wu, T., Zafonte, R., Valadka, A. B., Okonkwo, D. O., ... Li, J. (2018). Sub-classifying patients with mild traumatic brain injury: A clustering approach based on baseline clinical characteristics and 90-day and 180-day outcomes. PLoS ONE, 13(7), 1-18. https://doi.org/10.1371/journal.pone.0198741

Van Horn, J. D., Bhattrai, A., \& Irimia, A. (2017). Multimodal Imaging of Neurometabolic Pathology due to Traumatic Brain Injury. Trends in Neurosciences. Elsevier Ltd. https://doi.org/10.1016/j.tins.2016.10.007

Vergara, V. M., Mayer, A. R., Kiehl, K. A., \& Calhoun, V. D. (2018). Dynamic functional network connectivity discriminates mild traumatic brain injury through machine learning. NeuroImage: Clinical, 19(March), 30-37. https://doi.org/10.1016/j.nicl.2018.03.017

Voormolen, D. C., Haagsma, J. A., Polinder, S., Maas, A. I. R., Steyerberg, E. W., Vuleković, P., ... von Steinbuechel, N. (2019). Post-Concussion Symptoms in 
Complicated vs. Uncomplicated Mild Traumatic Brain Injury Patients at Three and Six Months Post-Injury: Results from the CENTER-TBI Study. Journal of Clinical Medicine, 8(11), 1921. https://doi.org/10.3390/jcm8111921

Walker, A. K., \& Dantzer, R. (2014). Is there a role for glutamate-mediated excitotoxicity in inflammation-induced depression? Journal of Neuroal Transmission, 121(8), 952-932. https://doi.org/10.1007/s00702-014-1187-1.Is

Wallace, E. J., Mathias, J. L., \& Ward, L. (2018). The relationship between diffusion tensor imaging findings and cognitive outcomes following adult traumatic brain injury: A meta-analysis. Neuroscience and Biobehavioral Reviews, 92(March), 93103. https://doi.org/10.1016/j.neubiorev.2018.05.023

Yi, J. H., \& Hazell, A. S. (2006). Excitotoxic mechanisms and the role of astrocytic glutamate transporters in traumatic brain injury. Neurochemistry International, 48(5), 394-403. https://doi.org/10.1016/j.neuint.2005.12.001

Yue, J. K., Vassar, M. J., Lingsma, H. F., Cooper, S. R., Okonkwo, D. O., Valadka, A. B., ... Sinha, T. K. (2013). Transforming research and clinical knowledge in traumatic brain injury pilot: Multicenter implementation of the common data elements for traumatic brain injury. Journal of Neurotrauma, 30(22), 1831-1844. https://doi.org/10.1089/neu.2013.2970

Yuh, E. L., Cooper, S. R., Mukherjee, P., Yue, J. K., Lingsma, H. F., Gordon, W. A., ... Sinha, T. K. (2014). Diffusion tensor imaging for outcome prediction in mild traumatic brain injury: a TRACK-TBI study. Journal of Neurotrauma, 31(17), 1457-1477. https://doi.org/10.1089/neu.2013.3171

Zimmermann, J., Perry, A., Breakspear, M., Schirner, M., Sachdev, P., Wen, W., ... Solodkin, A. (2018). Differentiation of Alzheimer's disease based on local and global parameters in personalized Virtual Brain models. NeuroImage: Clinical, 19(April), 240-251. https://doi.org/10.1016/j.nicl.2018.04.017 
Figures and Tables

881 Table 1. Patient Characteristics

\begin{tabular}{|c|c|c|c|c|c|}
\hline Scale & Subscale & $\begin{array}{l}\text { CT/MRI positive } \\
\mathrm{n}=14\end{array}$ & $\begin{array}{l}\text { CT/MRI negative } \\
\mathrm{n}=30\end{array}$ & $\begin{array}{l}\text { Comparison } \\
\text { participants } \\
n=36\end{array}$ & $\begin{array}{l}\text { Analysis for group } \\
\text { difference* }\end{array}$ \\
\hline Age & & $39.9(13.8)$ & $31.2(9.0)$ & $26.6(7.7)$ & $\mathrm{F}(2,79)=9.7, \mathrm{p}=0.0001$ \\
\hline Gender & & $\begin{array}{l}9 \text { Male; } 5 \text { Female } \\
9 \text { Caucasian, } 2 \text { More } \\
\text { than one race; } 1 \\
\text { African-American or } \\
\text { African; } 1 \text { Asian; } 1 \\
\text { Hawaiian or Pacific }\end{array}$ & $\begin{array}{l}18 \text { Male; } 12 \text { Female } \\
23 \text { Caucasian; } 3 \text { Asian; } \\
2 \text { Hawaiian or Pacific } \\
\text { Islander; } 1 \text { African } \\
\text { American or African; } 1\end{array}$ & $\begin{array}{l}25 \text { Male; } 11 \text { Female } \\
\text { Unknown }\end{array}$ & $X(2)^{2}=0.65, p=0.72$ \\
\hline Race & & Islander & More than one race & & \\
\hline Education & & $14.6(2.1)$ & $14.6(3.1)$ & Unknown & $\mathrm{t}(42)=-0.08, \mathrm{p}=0.93$ \\
\hline Glasgow Coma Scale & & $14.6(0.63)$ & $14.9(0.43)$ & $\mathrm{Na}$ & $\mathrm{t}(42)=1.4, \mathrm{p}=0.18$ \\
\hline Loss of Consciousness & & $\begin{array}{l}8 \text { None; } 6<0.5 \text { Hours } \\
5 \text { None } 4<0.5 \text { Hours; } 5\end{array}$ & $\begin{array}{l}12 \text { None; } 18<0.5 \text { Hours } \\
14 \text { None; } 15<0.5\end{array}$ & $\begin{array}{l}\mathrm{Na} \\
\mathrm{Na}\end{array}$ & $\begin{array}{l}X(1)^{2}=0.55, p=0.46 \\
X(1)^{2}=8.6, p=0.01\end{array}$ \\
\hline Post Traumatic Amnesia & & $\begin{array}{l}0.5-24 \text { Hours } \\
n=11\end{array}$ & $\begin{array}{l}\text { Hours; } 10.5 \text {-24 Hours } \\
n=27\end{array}$ & na & \\
\hline $\begin{array}{l}\text { Glasgow Outcome Scale } \\
\text { Extended } \\
\text { Brief Symptom }\end{array}$ & 6 month & $6.8(0.98)$ & $7.0(0.94)$ & na & $\mathrm{t}(36)=0.64, \mathrm{p}=0.52$ \\
\hline Inventory & $\begin{array}{l}\text { Anxiety } \\
\text { Depression } \\
\text { Somatic } \\
\text { Global Severity Index }\end{array}$ & $\begin{array}{l}54.9(7.7) \\
54.2(10.2) \\
55.8(7.9) \\
57.0(7.3)\end{array}$ & $\begin{array}{l}53.6(10.6) \\
52.6(10.6) \\
53.0(9.7) \\
53.6(10.8)\end{array}$ & $\begin{array}{l}\text { na } \\
\text { na } \\
\text { na } \\
\text { na }\end{array}$ & $\begin{array}{l}\mathrm{t}(36)=-0.37, \mathrm{p}=0.71 \\
\mathrm{t}(36)=-0.42, \mathrm{p}=0.67 \\
\mathrm{t}(36)=-0.86, \mathrm{p}=0.39 \\
\mathrm{t}(36)=-0.97, \mathrm{p}=0.34\end{array}$ \\
\hline & & $\mathrm{n}=11$ & $\mathrm{n}=26$ & na & \\
\hline $\begin{array}{l}\text { Satisfaction with life } \\
\text { score }\end{array}$ & & $\begin{array}{l}19.1(7.6) \\
n=11\end{array}$ & $\begin{array}{l}22.6(5.9) \\
n=25\end{array}$ & $\begin{array}{l}\text { na } \\
\text { na }\end{array}$ & $\mathrm{t}(35)=1.5, \mathrm{p}=0.14$ \\
\hline $\begin{array}{l}\text { Trail Making Test } \\
\text { Wechsler Adult }\end{array}$ & $\begin{array}{l}\text { Part A } \\
\text { Part B } \\
\text { Processing Speed }\end{array}$ & $\begin{array}{l}30.7(9.7) \\
69.8(25.0) \\
109.2(16.3)\end{array}$ & $\begin{array}{l}28.3(10.6) \\
76.4(65.2)\end{array}$ & $\begin{array}{l}\text { na } \\
\text { na }\end{array}$ & $\begin{array}{l}t(35)=-0.65, p=0.52 \\
t(35)=0.32, p=0.75\end{array}$ \\
\hline
\end{tabular}




$106.5(14.6)$

*The following statistics are reported: One-way ANOVA (age), Chi square test of independence (gender, loss of consciousness, posttraumatic amnesia), independent samples t test (Education, Glasgow Coma Scale, Glasgow Outcome Scale Extended (6 month), Brief Symptom Inventory, Satisfaction with Life Score, Trail Making Test Part A and B, Wechsler Adult Intelligence Scale, and California Verbal Learning Test.

Table 2. MRI Radiological Findings of the CT/MRI Positive TBI Group

14 contusions, 1 shear (MRI)

2 shear (MRI)

1 shear (MRI)

2 shear (MRI)

4 contusions (MRI)

1 intracranial lesions, 1 skull fracture, 1 subdural hematoma, 1 contusion, 1 brain swelling (CT)

4 contusions, 3 shears (MRI)

2 shear (MRI)

1 skull fracture $(\mathrm{CT})$

1 contusion, 2 shear (MRI)

1 intracranial lesions, 1 sub arachnoid haemorrhage, 1 contusion

101 subdural hematoma, 2 contusions (MRI)

1 intracranial lesions, 1 subarachnoid haemorrhage, 1 contusion

111 subdural hematoma, 2 contusions, 2 shear (MRI)

1 intracranial lesions, 1 skull fracture, 1 subdural hematoma, 1 subarachnoid hematoma

121 intracranial lesions, 1 subarachnoid hematoma (CT)

132 shear (MRI) 
889 Table 3. Cortical and subcortical regions from the Regional Map parcellation from Kötter 890 \& Wanke (2005)

\begin{tabular}{|c|c|c|}
\hline \multicolumn{2}{|l|}{ Index } & \multirow[b]{2}{*}{ Region } \\
\hline Right & Left & \\
\hline 8 & 49 & primary auditory cortex \\
\hline 2 & 50 & secondary auditory cortex \\
\hline 3 & 51 & amygdala \\
\hline 4 & 52 & anterior cingulate cortex \\
\hline 5 & 53 & posterior cingulate cortex \\
\hline 6 & 54 & retrosplenial cingulate cortex \\
\hline 7 & 55 & subgenual cingulate cortex \\
\hline 8 & 56 & frontal eye field \\
\hline 9 & 57 & gustatory cortex \\
\hline 10 & 58 & hippocampus \\
\hline 11 & 59 & anterior insula \\
\hline 12 & 60 & posterior insula \\
\hline 13 & 61 & primary motor cortex \\
\hline 14 & 62 & inferior parietal cortex \\
\hline 15 & 63 & intraparietal cortex \\
\hline 16 & 64 & medial parietal cortex \\
\hline 17 & 65 & superior parietal cortex \\
\hline 18 & 66 & centrolateral prefrontal cortex \\
\hline 19 & 67 & dorsolateral prefrontal cortex \\
\hline 20 & 68 & dorsomedial prefrontal cortex \\
\hline 21 & 69 & medial prefrontal cortex \\
\hline 22 & 70 & orbitoinferior prefrontal cortex \\
\hline 23 & 71 & orbitolateral prefrontal cortex \\
\hline 24 & 72 & orbitomedial prefrontal cortex \\
\hline 25 & 73 & prefrontal polar cortex \\
\hline 26 & 74 & ventrolateral prefrontal cortex \\
\hline 27 & 75 & parahippocampal cortex \\
\hline 28 & 76 & dorsolateral premotor cortex \\
\hline 29 & 77 & medial premotor cortex \\
\hline 30 & 78 & ventrolateral premotor cortex \\
\hline 31 & 79 & primary somatosensory cortex \\
\hline 32 & 80 & secondary somatosensory cortex \\
\hline 33 & 81 & central temporal cortex \\
\hline 34 & 82 & inferior temporal cortex \\
\hline 35 & 83 & temporal polar cortex \\
\hline 36 & 84 & superior temporal cortex \\
\hline 37 & 85 & ventral temporal cortex \\
\hline 38 & 86 & visual area 1 (primary visual cortex) \\
\hline 39 & 87 & visual area 2 (secondary visual cortex) \\
\hline 40 & 88 & anterior visual area, dorsal part \\
\hline 41 & 89 & anterior visual area, ventral part \\
\hline 42 & 90 & thalamic roi with major frontal connections \\
\hline 43 & 91 & thalamic roi with major temporal connections \\
\hline 44 & 92 & thalamic roi with major occipitoparietal connections \\
\hline 45 & 93 & caudate nucleus \\
\hline 46 & 94 & putamen \\
\hline 47 & 95 & pallidum \\
\hline 48 & 96 & accumbens nucleus \\
\hline
\end{tabular}


891 Table 4. TVB model parameters

\begin{tabular}{|c|c|c|}
\hline Parameter & $\begin{array}{l}\text { Value (number } \\
\text { of steps) }\end{array}$ & Description \\
\hline $\begin{array}{l}\text { Global Coupling } \\
(G)\end{array}$ & $1.4-2.8(50)$ & $\begin{array}{l}\text { scaling factor for inter-region (global) } \\
\text { excitatory coupling }\end{array}$ \\
\hline Noise $(\sigma)$ & 0.001 & amplitude of noise kernel \\
\hline $\begin{array}{l}\text { Conduction } \\
\text { Velocity }(\mathrm{m} / \mathrm{s})\end{array}$ & 6 & $\begin{array}{l}\text { speed of inter-region (global) signal } \\
\text { transmission }\end{array}$ \\
\hline $\mathrm{W}_{+}$ & 1.4 & Excitatory recurrent potential \\
\hline $\mathrm{J}_{\mathrm{GABA}}(\mathrm{nA})$ & $1.0 *$ & Local feedback inhibitory synaptic coupling \\
\hline $\mathrm{J}_{\mathrm{NMDA}}(\mathrm{nA})$ & 0.15 & Local excitatory coupling \\
\hline Time steps (ms) & 600000 & Simulation duration \\
\hline fMRI TR (ms) & 2000 & Simulation repetition time (TR) \\
\hline
\end{tabular}

894 Table 5. Factor loadings for 6-month outcome variables

\begin{tabular}{llrr}
\hline Scale & Subscale & TBI Symptoms & Age and Cognition \\
\hline Glasgow Outcome Scale Extended & 6 month & $-\mathbf{0 . 7 3}$ & -0.033 \\
Brief Symptom Inventory & Somatic & $\mathbf{0 . 7 1}$ & 0.16 \\
& Depression & $\mathbf{0 . 8 6}$ & -0.075 \\
& Anxiety & $\mathbf{0 . 8 7}$ & -0.032 \\
& Global severity index & $\mathbf{0 . 9 9}$ & -0.043 \\
Satisfaction with life scale & & $-\mathbf{0 . 8 3}$ & 0.29 \\
Education & & $-\mathbf{0 . 3 3}$ & $\mathbf{- 0 . 5 1}$ \\
Age & & -0.036 & $\mathbf{0 . 4 2}$ \\
Trail Making Test & Part A & 0.19 & $\mathbf{0 . 6 2}$ \\
& Part B & -0.085 & $\mathbf{0 . 8 9}$ \\
Wechsler Adult Intelligence Scale & Processing Speed & 0.25 & $-\mathbf{0 . 9 2}$ \\
California Verbal Learning Test & & -0.10 & $\mathbf{- 0 . 3 1}$ \\
Percent Covariance & & $37 \%$ & $22 \%$ \\
\hline
\end{tabular}

895 *loadings $>|0.3|$ are bolded to assist interpretation

$896 * *$ Note: BSI and TMT scales are reverse coded such that higher scores indicate more 897 symptoms or poorer performance

898 Table 6. Model fitting results

\begin{tabular}{|c|c|c|c|c|c|}
\hline Fitting Metric & $\begin{array}{l}\text { Descriptive } \\
\text { statistic }\end{array}$ & $\begin{array}{l}\text { CT/MRI } \\
\text { positive }\end{array}$ & $\begin{array}{l}\mathrm{CT} / \mathrm{MRI} \\
\text { negative }\end{array}$ & Comparison & Significance \\
\hline & & $n=14$ & $\mathbf{n}=\mathbf{3 0}$ & $n=36$ & \\
\hline Functional & mean & 0.66 & 0.66 & 0.68 & \multirow[t]{4}{*}{$\mathrm{F}(2,77)=0.32, \mathrm{p}=0.73$} \\
\hline $\begin{array}{l}\text { Connectivity, } \\
\text { uncentered }\end{array}$ & $\begin{array}{l}\text { standard } \\
\text { deviation }\end{array}$ & 0.11 & 0.09 & 0.12 & \\
\hline correlation & minimum & 0.50 & 0.33 & 0.35 & \\
\hline & maximum & 0.79 & 0.84 & 0.88 & \\
\hline Functional & mean & 0.13 & 0.12 & 0.12 & \multirow[t]{3}{*}{$\mathrm{F}(2,77)=0.03, \mathrm{p}=0.97$} \\
\hline $\begin{array}{l}\text { connectivity } \\
\text { dynamics, }\end{array}$ & $\begin{array}{l}\text { standard } \\
\text { deviation }\end{array}$ & 0.07 & 0.14 & 0.13 & \\
\hline Kolmogorov- & minimum & 0.04 & 0.03 & 0.03 & \\
\hline
\end{tabular}




\begin{tabular}{|c|c|c|c|c|c|}
\hline $\begin{array}{l}\text { Smirnov } \\
\text { distance }\end{array}$ & maximum & 0.31 & 0.70 & 0.74 & \\
\hline Percentage of & mean & $37.0 \%$ & $34.5 \%$ & $34.3 \%$ & $F(2,77)=0.15, p=0.86$ \\
\hline $\begin{array}{l}\text { iterations } \\
\text { optimal }\end{array}$ & $\begin{array}{l}\text { standard } \\
\text { deviation }\end{array}$ & $13.7 \%$ & $16.2 \%$ & $14.9 \%$ & \\
\hline solution was & minimum & $15.0 \%$ & $15.0 \%$ & $15.0 \%$ & \\
\hline chosen for & maximum & $75.0 \%$ & $90.0 \%$ & $90.0 \%$ & \\
\hline
\end{tabular}

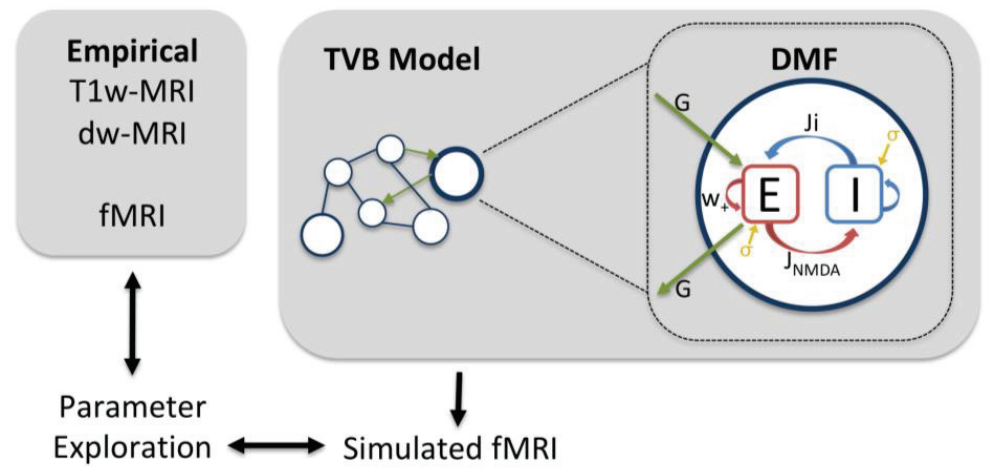

901 Figure 1. The Virtual Brain workflow. Structural and functional connectomes were 902 created from each subject's dwMRI and fMRI data respectively. Each subject's unique structural connectome constrained their personal brain simulation, wherein local dynamics were represented by the dynamic mean field (DMF) model (equations 1-6) (Deco, McIntosh, et al., 2014; Deco, Ponce-Alvarez, et al., 2014). The simulated local patic gatentials were then fed through the Balloon-Windkessel hemodynamic model, producing simulated fMRI timeseries. Each subject's simulated fMRI timeseries was fitted to their functional connectome through parameter space exploration. The resulting subject-specific parameters were used in later analyses. Abbreviations: Dynamic Mean Field model (DMF), excitatory neural population (E), inhibitory neural population (I), global coupling $(G)$, recurrent potential $\left(w_{+}\right)$, inhibitory 

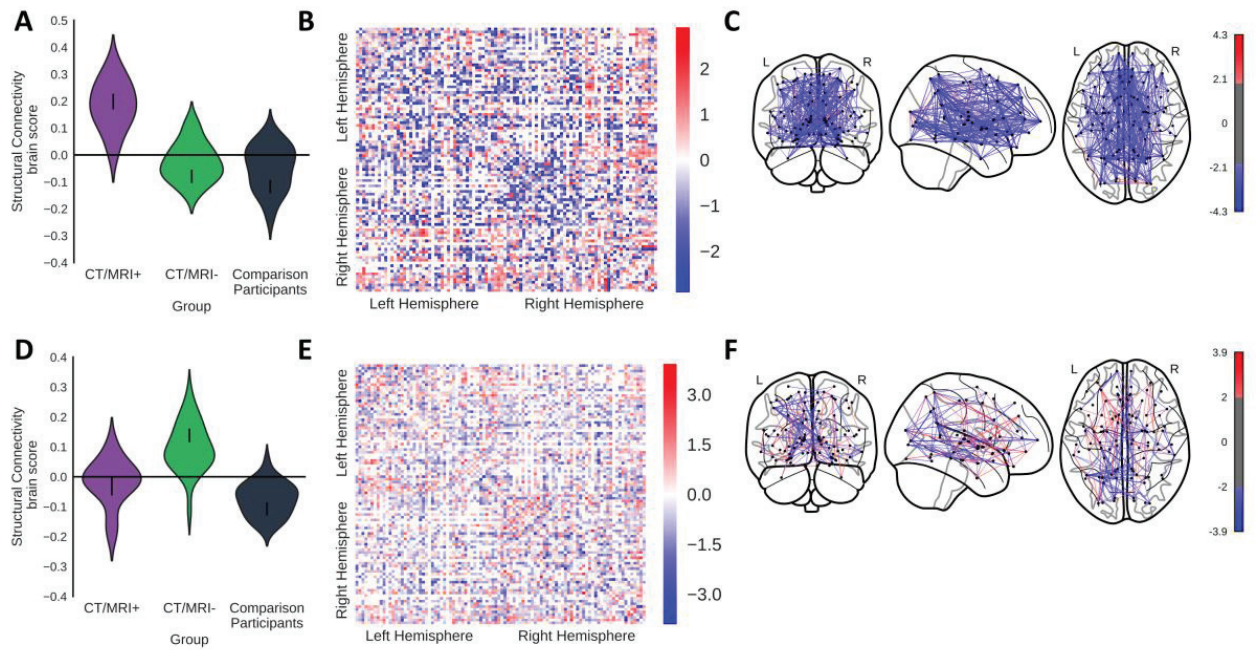

$\mathrm{E}$
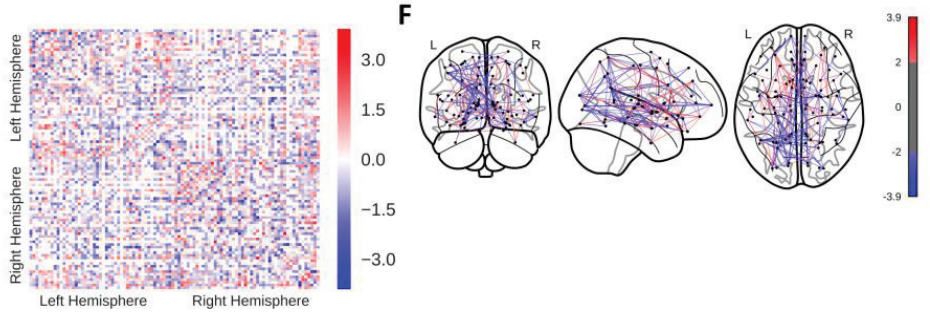

914

915

916

917

918

919

920

921

922

923

924

925

926

927

Figure 2. A group comparison PLS distinguished the SC of CT/MRI positive and negative patients from comparison participants. A-C Shows the first latent variable $(p<0.0001,67 \%$ covariance, singular value $=0.19)$ that differentiated CT/MRI positive patients from the comparison participants, while D-F Shows the second latent variable $(p=0.016,33 \%$ covariance, singular value $=0.13)$ that distinguished CT/MRI negative patients from the comparison participants. A/D Violin plots show the distribution of brain scores for each group. Brain scores indicate the degree to which participants express the pattern of SC shown in 'B'/'E'. Error bars are bootstrap-estimated 95\% confidence intervals. B/E Show bootstrap ratios, which are a linear combination of SC weighted by how strongly they contribute to the latent variable. Bootstrap ratios may be interpreted similar to $\mathrm{Z}$-score $(>|2.0|$ akin to $p<0.05)$, so regions with bars exceeding the dashed line may be considered reliably contributing to the latent variable. C/F Regional inhibitory connection strength bootstrap ratios that are reliably contributing to the latent variable $(>|2|)$ from 'B'/'E' projected onto a brain.

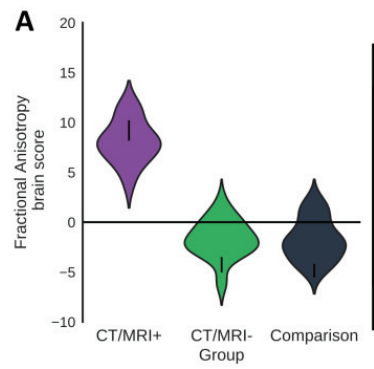

\section{B}

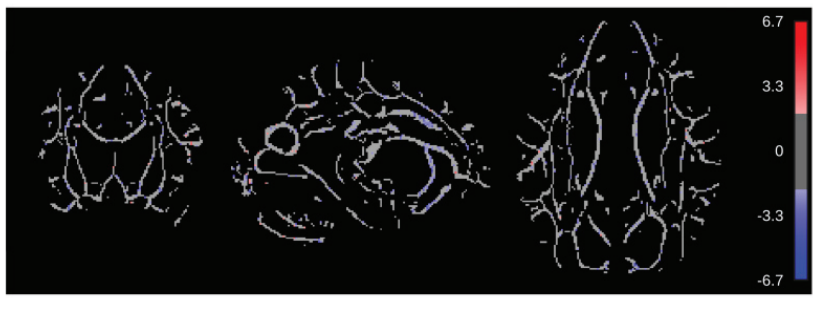

Figure 3. A group comparison PLS distinguished the CT/MRI positive and negative patients from comparison participants via their fractional anisotropy. A-B Shows the first latent variable $(p=0.01,65 \%$ covariance, singular value $=8.2)$ that distinguished $\mathrm{CT} / \mathrm{MRI}$ positive patients from the comparison participants A Violin plot shows the distribution of 
brain scores for each group. Brain scores indicate the degree to which participants express the pattern fractional anisotropy shown in 'B'. Error bars are bootstrap-estimated $95 \%$ confidence intervals. B Shows bootstrap ratios, which are a linear combination of voxel-wise fractional anisotropy weighted by how strongly they contribute to the latent variable. Bootstrap ratios are superimposed onto a white matter skeleton. Bootstrap ratios may be interpreted similar to z-score $(>|2.0|$ akin to $p<0.05)$, so only voxels with bootstrap ratios exceeding $|2|$ are illustrated.

A

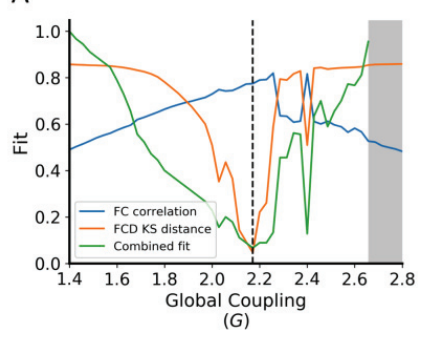

B

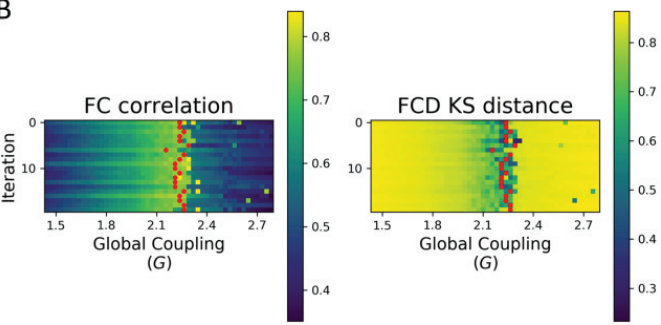

Figure 4. Summary of modeling fitting procedure. A The parameter space exploration map for a representative subject given a single iteration. The gray area denotes values of global coupling for which the model fails to converge because it becomes hyperexcited. The black dashed line represents the optimal value of global coupling. Note that combined fit (green) is defined by the sum of the FC and FCD fits ranked across all other values of global coupling at which the model converged. B Shows the full parameter space results for the same representative subject. Each grid shows model fits across all values of $G$ on the x-axis, and iterations with randomized initial conditions on the y-axis. On the left, fits are defined by the uncentered correlation of the upper triangle of the empirical and simulated FC matrices. On the right, Kolmogorov-Smirnov (KS) distance between the upper triangles of the empirical and simulated FCD matrices defines fits. The red dots represent the optimal fit for each iteration. 

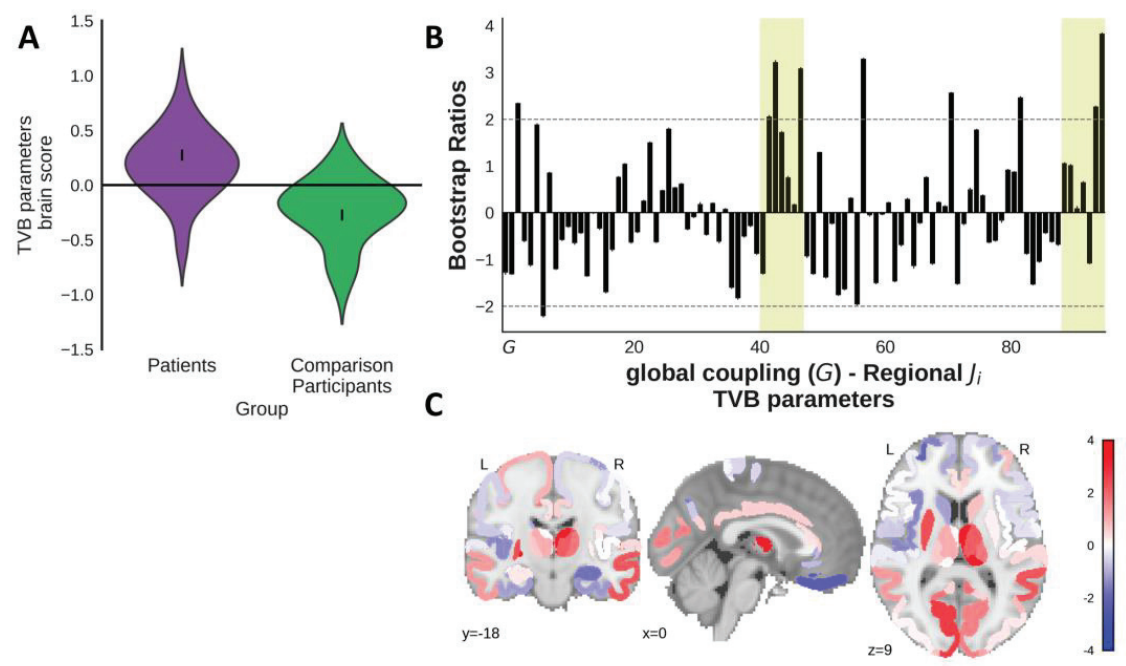

954 Figure 5. Group comparison PLS of TVB parameters (global coupling [G] and regional 955 inhibitory connection strengths) across patients (combined CT/MRI positive and negative subgroups) and comparison participants $(p=0.026$, singular value $=0.30)$. Patients showed mostly higher inhibitory connection strength relative to comparison participants, particularly in the subcortical regions. A The violin plot shows the distribution of brain scores for each group. Brain scores indicate the degree to which participants express the pattern of global coupling and regional local inhibitory connection strength shown in 'B'. Error bars are bootstrap-estimated $95 \%$ confidence intervals. B Shows bootstrap ratios, which are a linear combination of global coupling and regional local inhibitory connection strength weighted by how strongly they contribute to the latent variable. 964 Bootstrap ratios may be interpreted similar to z-score $(>|2.0|$ akin to $p<0.05)$, so regions with bars exceeding the dashed line may be considered reliably contributing to the latent variable. Error bars are one standard error. Bars representing subcortical regions are shaded. C Regional inhibitory connection strength bootstrap ratios from ' $\mathrm{B}$ ' projected onto a glass brain.

969 Note: $J_{\mathrm{i}}=$ inhibitory connection strength 


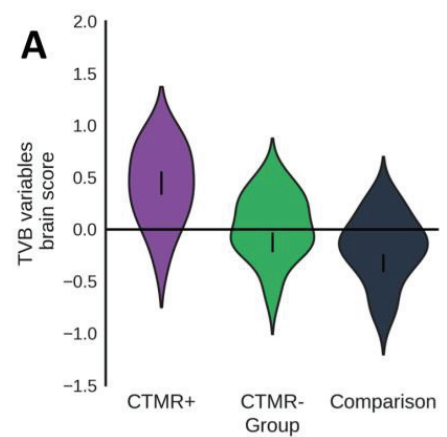

970

971

972

973

974

975

976

977

978

979

980

981

982

983

984

985
B

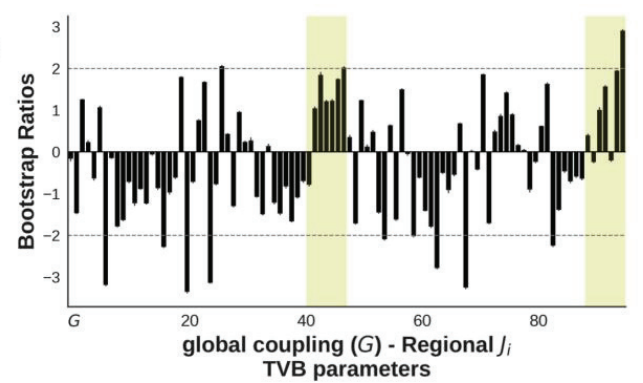

TVB parameters

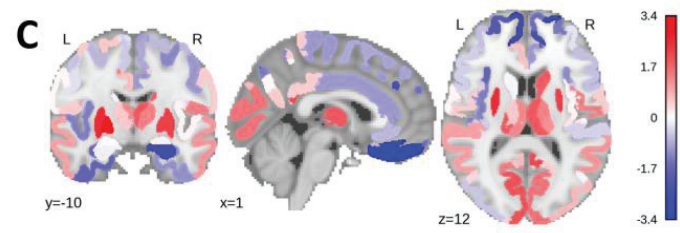

Figure 6. Group comparison PLS of TVB parameters (global coupling [G] and regional inhibitory connection strengths) across CT/MRI positive patients, CT/MRI negative patients, and comparison participants. A-C Shows the first latent variable $(p=0.03,70.9 \%$ covariance, singular value $=0.43$ ) that differentiated CT/MRI positive patients from comparison participants A Violin plot shows the distribution of brain scores for each group. Brain scores indicate the degree to which participants express the pattern of global coupling and regional local inhibitory connection strength shown in in 'B'. Error bars are bootstrap-estimated $95 \%$ confidence intervals. B Shows bootstrap ratios, which are a linear combination of global coupling and regional local inhibitory connection strength weighted by how strongly they contribute to the latent variable. Bootstrap ratios may be interpreted similar to z-score $(>|2.0|$ akin to $p<0.05)$, so regions with bars exceeding the dashed line may be considered reliably contributing to the latent variable. Error bars are one standard error. Bars representing subcortical regions are shaded. $\mathbf{C}$ Regional inhibitory connection strength bootstrap ratios from ' $\mathrm{B}$ ' projected onto a brain.

Note: $J_{\mathrm{i}}=$ inhibitory connection strength 


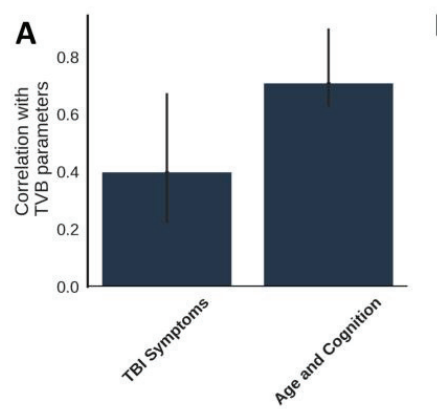

C
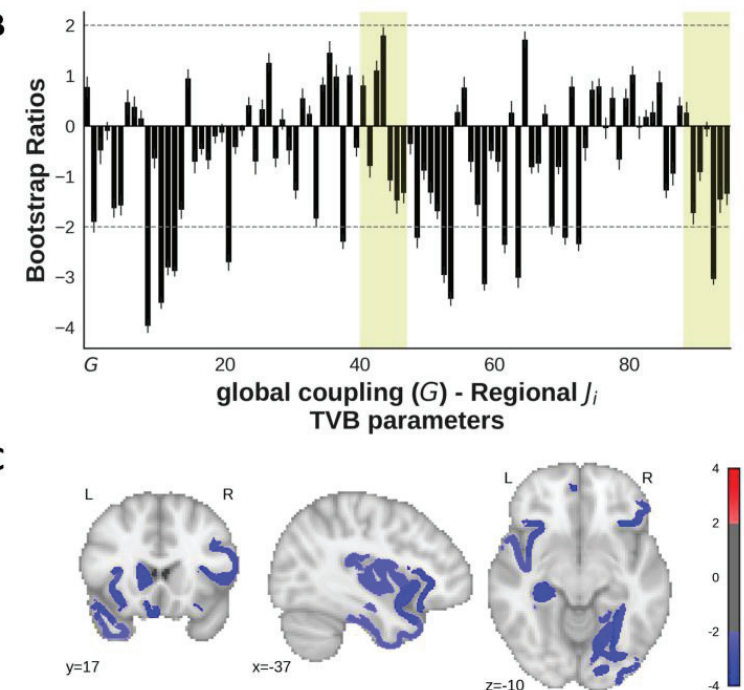

986

987

988

989

990

991

992

993

994

995

996

997

998

999

1000

1001

1002

Figure 7. A behavioural PLS analysis assessed the associations between the TBI Symptoms and Age and Cognition factors and TVB parameters (global coupling and regional inhibitory connection strengths) in the patients (combined CT/MRI positive and negative subgroups). The first significant variable is illustrated $(p=0.02,74 \%$ covariance, singular value=2.4) A The bars represent the correlation between each factor and the pattern of TVB parameters shown in the corresponding bar graph 'B'. The error bars represent $95 \%$ confidence intervals, so the error bars of variables significantly contributing to the latent variable do not cross zero. B Bootstrap ratios, which are a linear combination of global coupling and regional local inhibitory connection strength weighted by how strongly they contribute to the latent variable. Bootstrap ratios may be interpreted similar to z-score $(>|2.0|$ akin to $p<0.05)$, so regions with bars exceeding the dashed line may be considered reliably contributing to the latent variable. Error bars are one standard error. Bars representing subcortical regions are shaded. $\mathbf{C}$ Regional inhibitory connection strength bootstrap ratios from ' $\mathrm{B}$ ' that are reliably contributing to the latent variable $(>|2|)$ projected onto a brain.

Note: $J_{\mathrm{i}}=$ inhibitory connection strength 

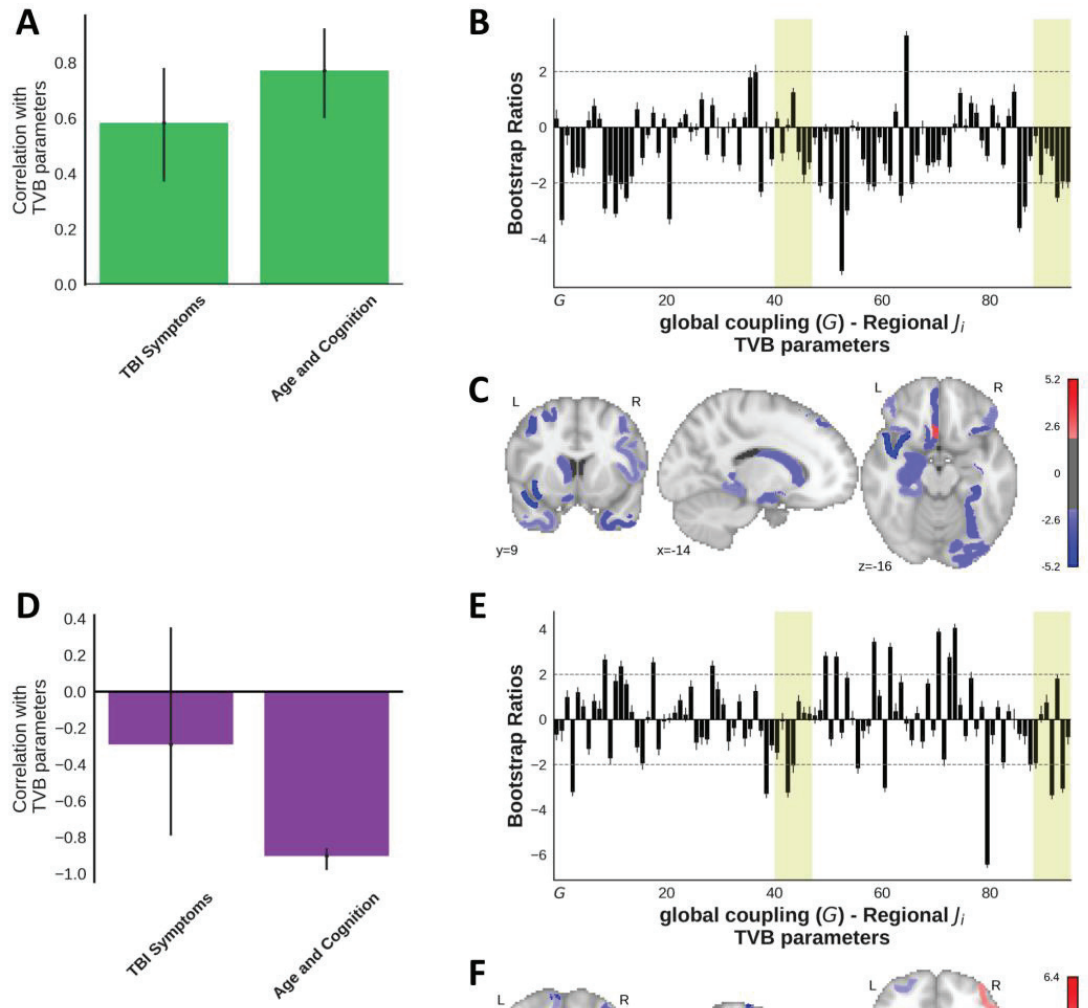

E
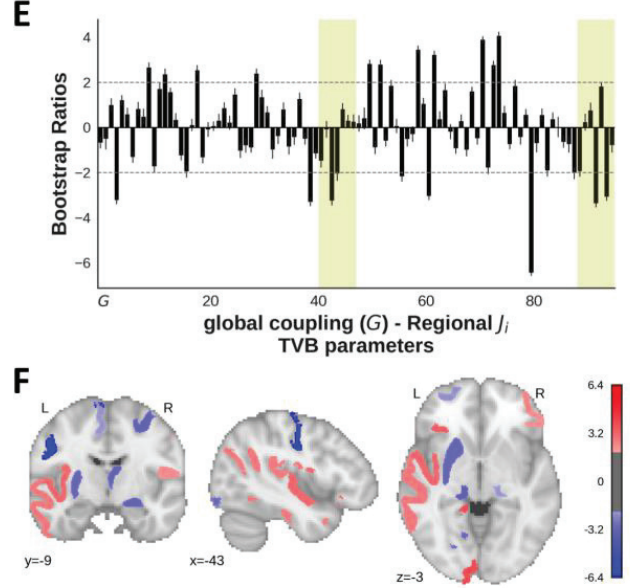

1003

1004

1005

1006

1007

1008

1009

1010

1011

1012

1013

1014

1015

1016

1017

1018

Figure 8. Within group behavioural PLS analyses show the relationships between the TBI Symptoms and Age and Cognition factor scores and the TVB parameters (global coupling $[\mathrm{G}]$ and regional inhibitory connection strengths) for the CT/MRI negative (AC) and CT/MRI positive patients (D-F). A-C Shows the first latent variable ( $p=0.005$, $73.1 \%$ covariance, singular value $=3.0$ ) for the within CT/MRI negative patients, while $\mathbf{D}$ F illustrates the first latent variable $(p=0.11,67.6 \%$ covariance, singular value $=3.7)$ for the CT/MRI positive patients. A/D The bars represent the correlation between each factor with the pattern of TVB parameters shown in the corresponding bar graph ' $\mathrm{B}$ '. The error bars represent $95 \%$ confidence intervals, so the error bars of variables significantly contributing to the latent variable do not cross zero. B/E Shows bootstrap ratios, which are a linear combination of global coupling and regional local inhibitory connection strength weighted by how strongly they contribute to the latent variable. Bootstrap ratios may be interpreted similar to $\mathrm{z}$-score $(>|2.0|$ akin to $p<0.05)$, so regions with bars exceeding the dashed line may be considered reliably contributing to the latent variable. Error bars are one standard error. Bars representing subcortical regions are shaded. $\mathbf{C} / \mathbf{F}$ 
1019 Regional inhibitory connection strength bootstrap ratios that are reliably contributing to 1020 the latent variable $(>|2|)$ from 'B'/'E' projected onto a brain.

1021 Note: $J_{\mathrm{i}}=$ inhibitory connection strength 


\section{Empirical} T1w-MRI dw-MRI

fMRI

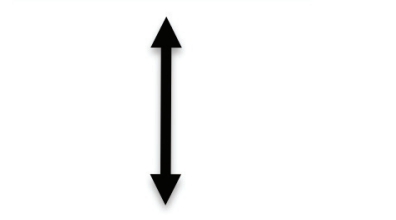

Parameter Exploration

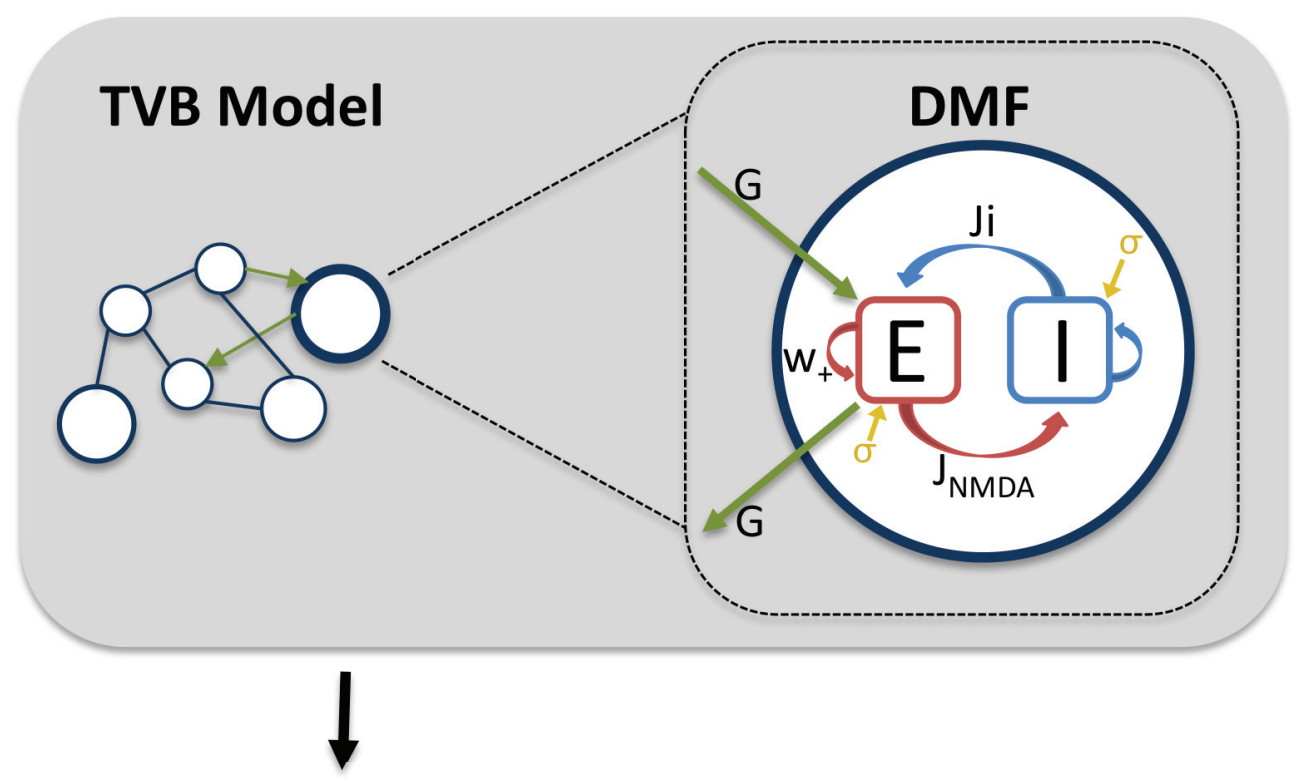

Simulated fMRI 

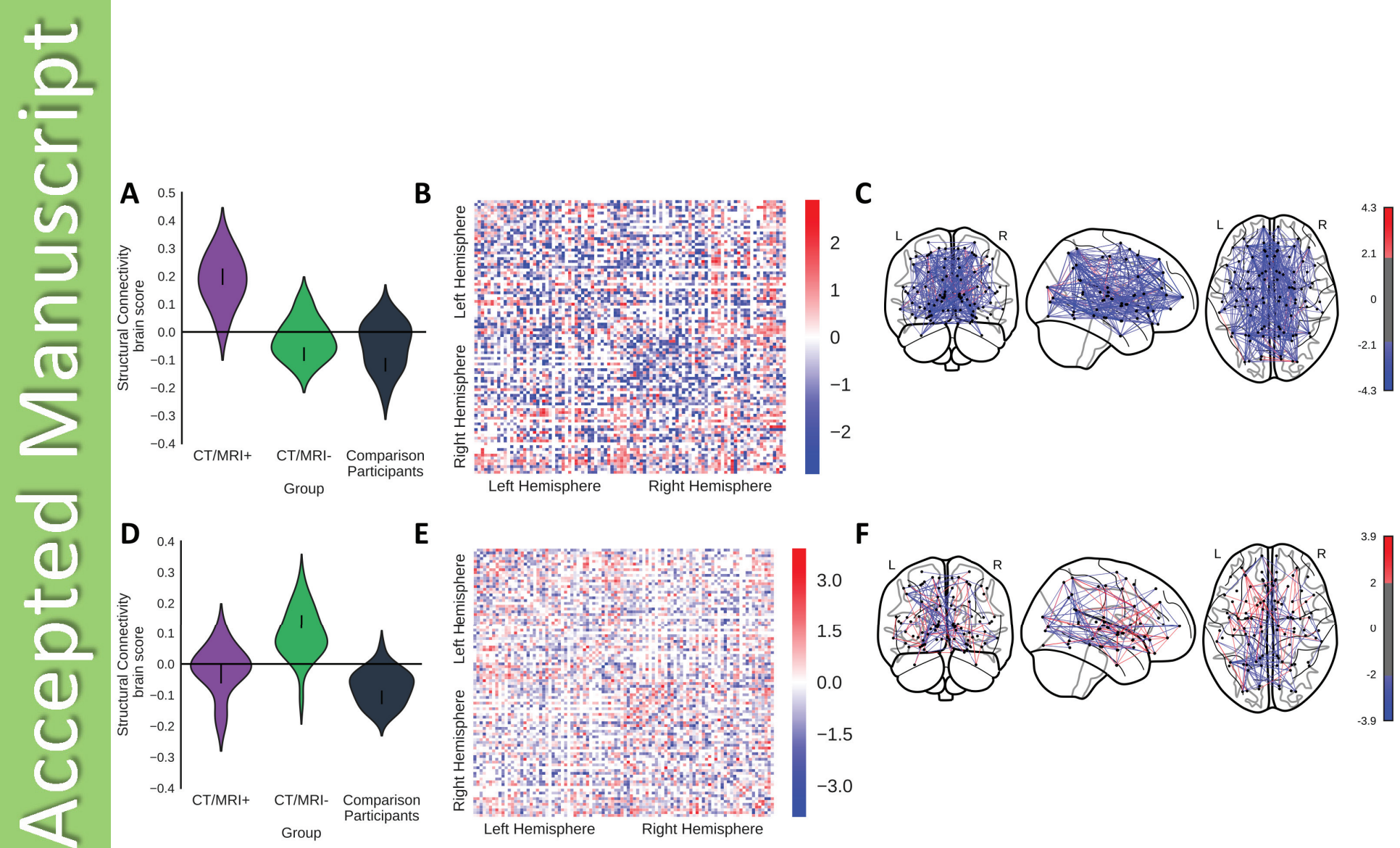

E

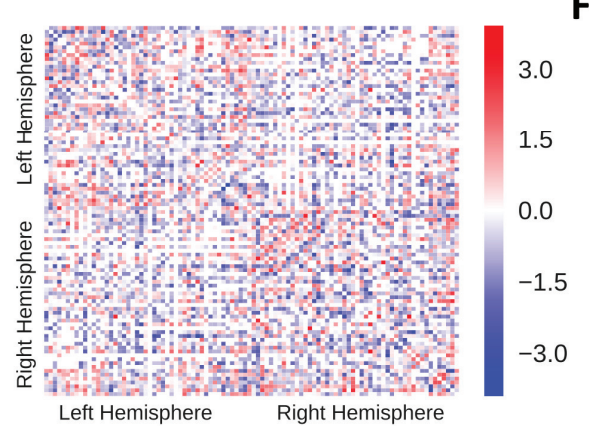

F

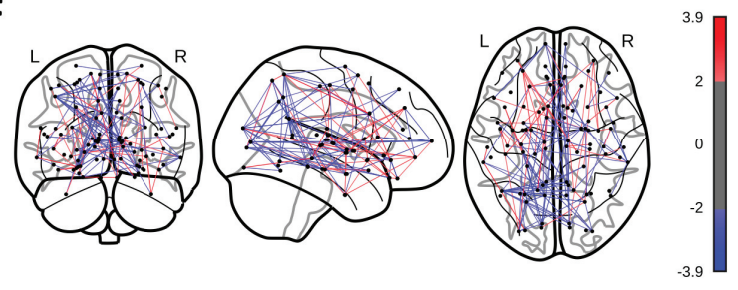

인 


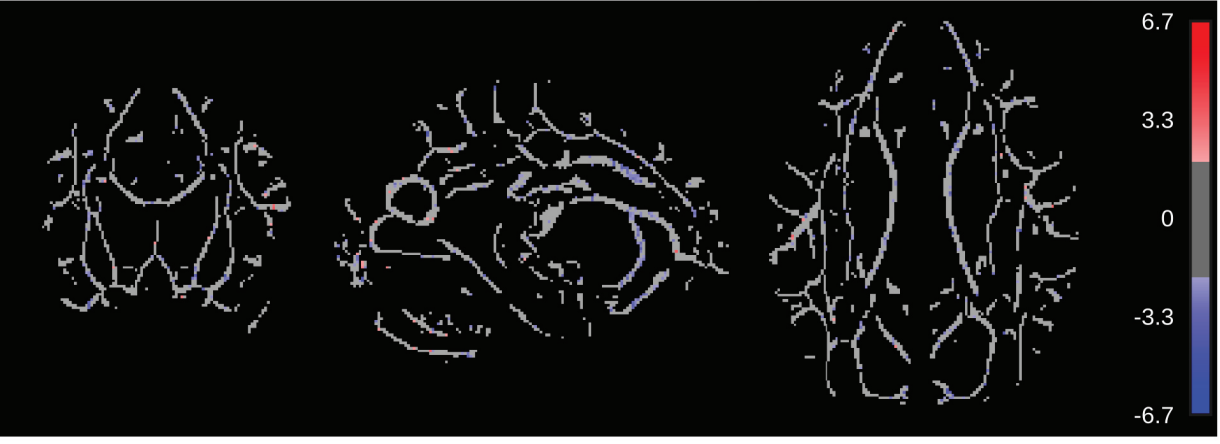

Group 
A

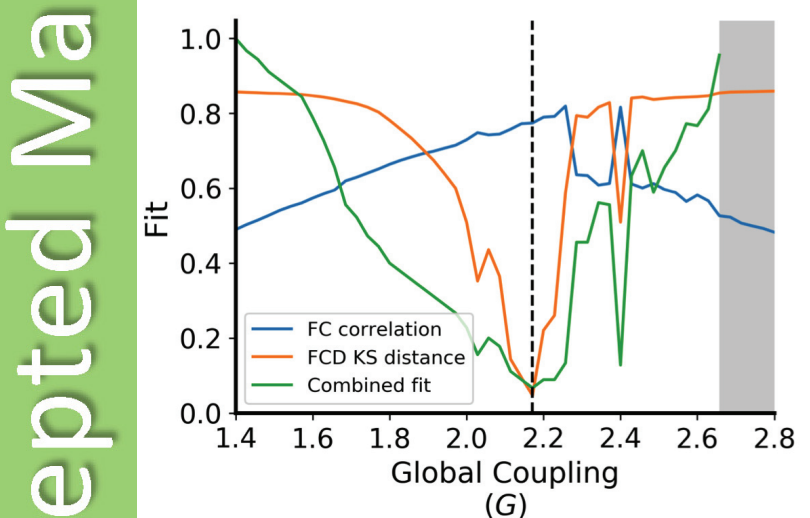

(G)

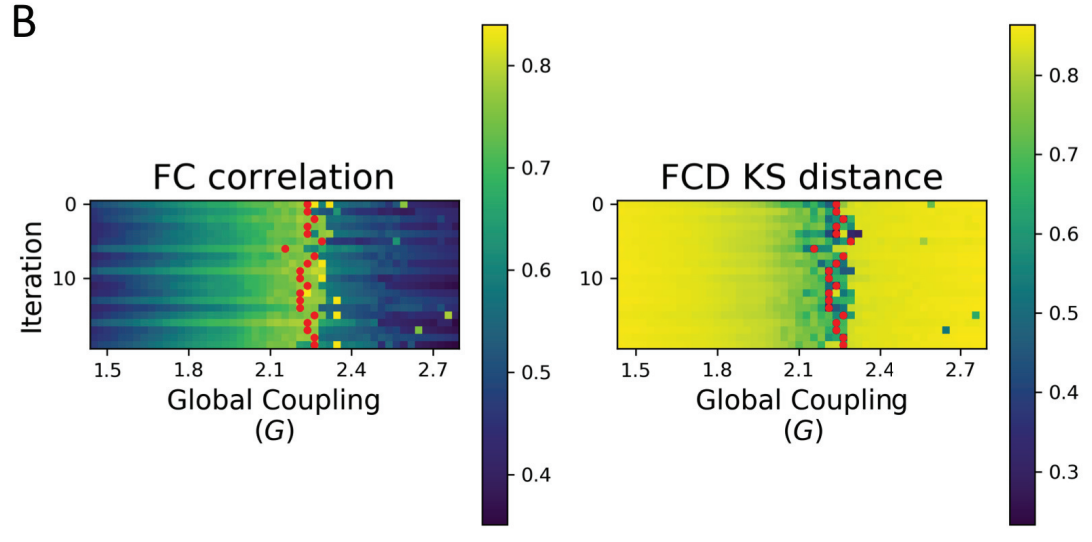



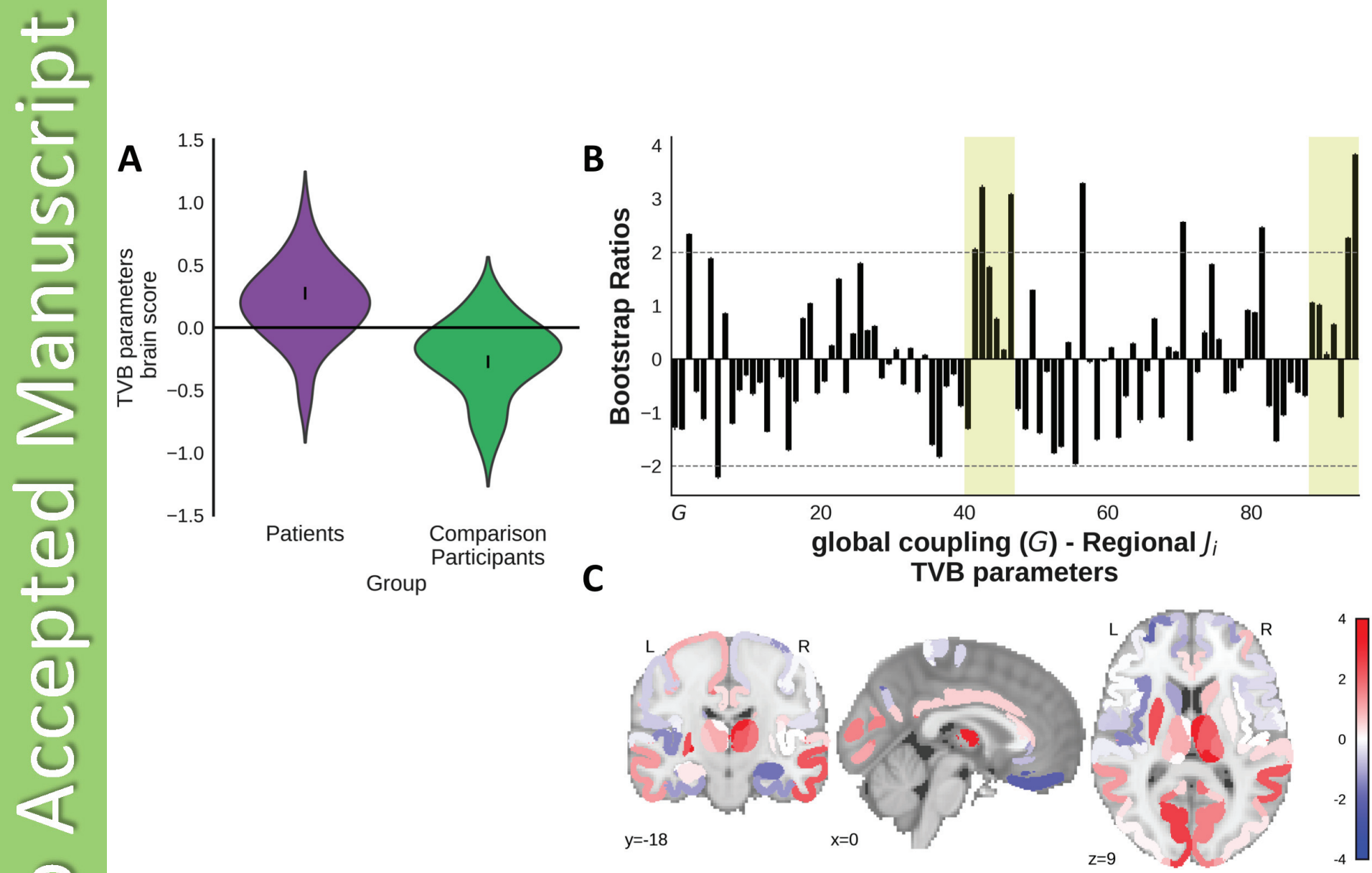

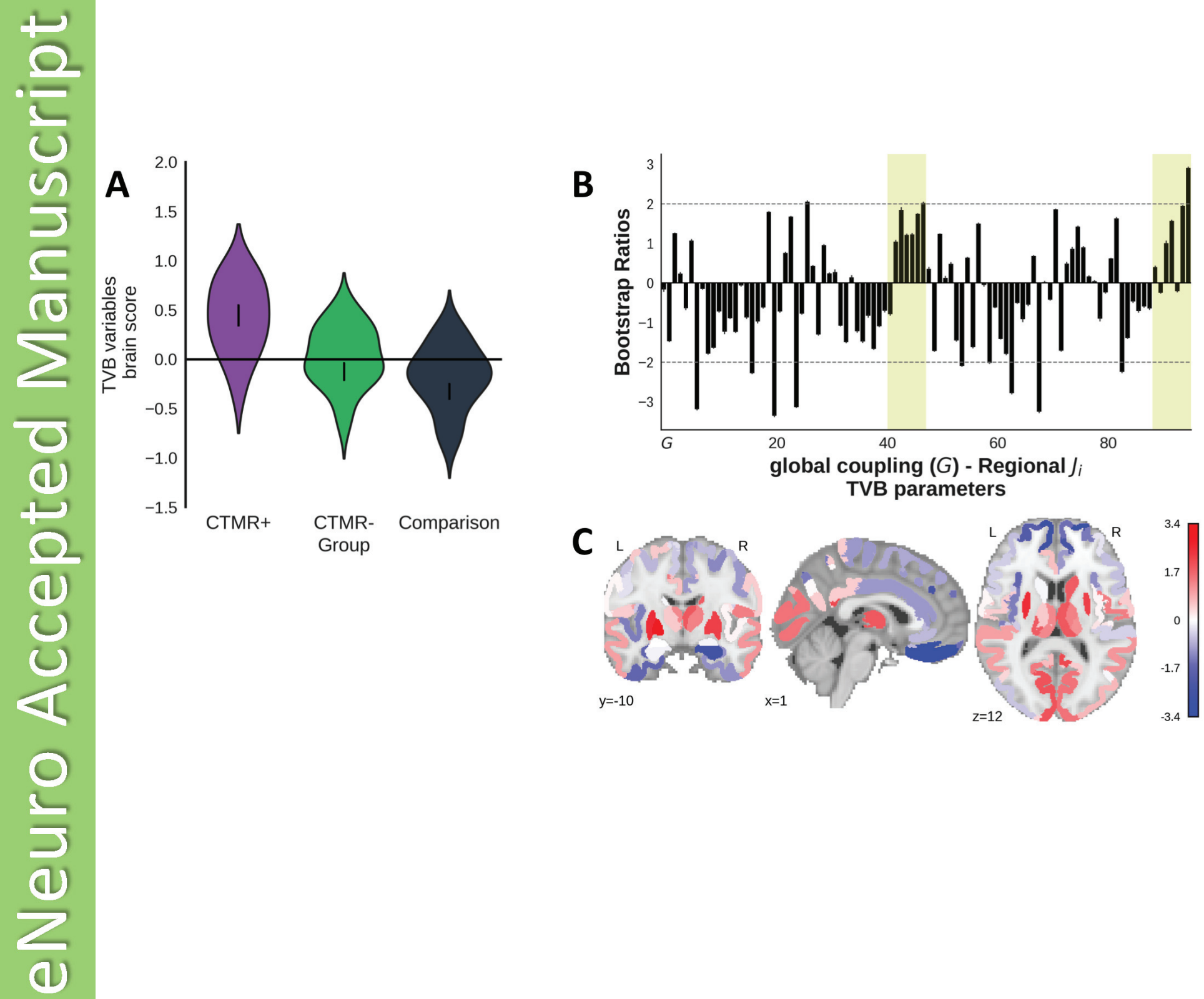

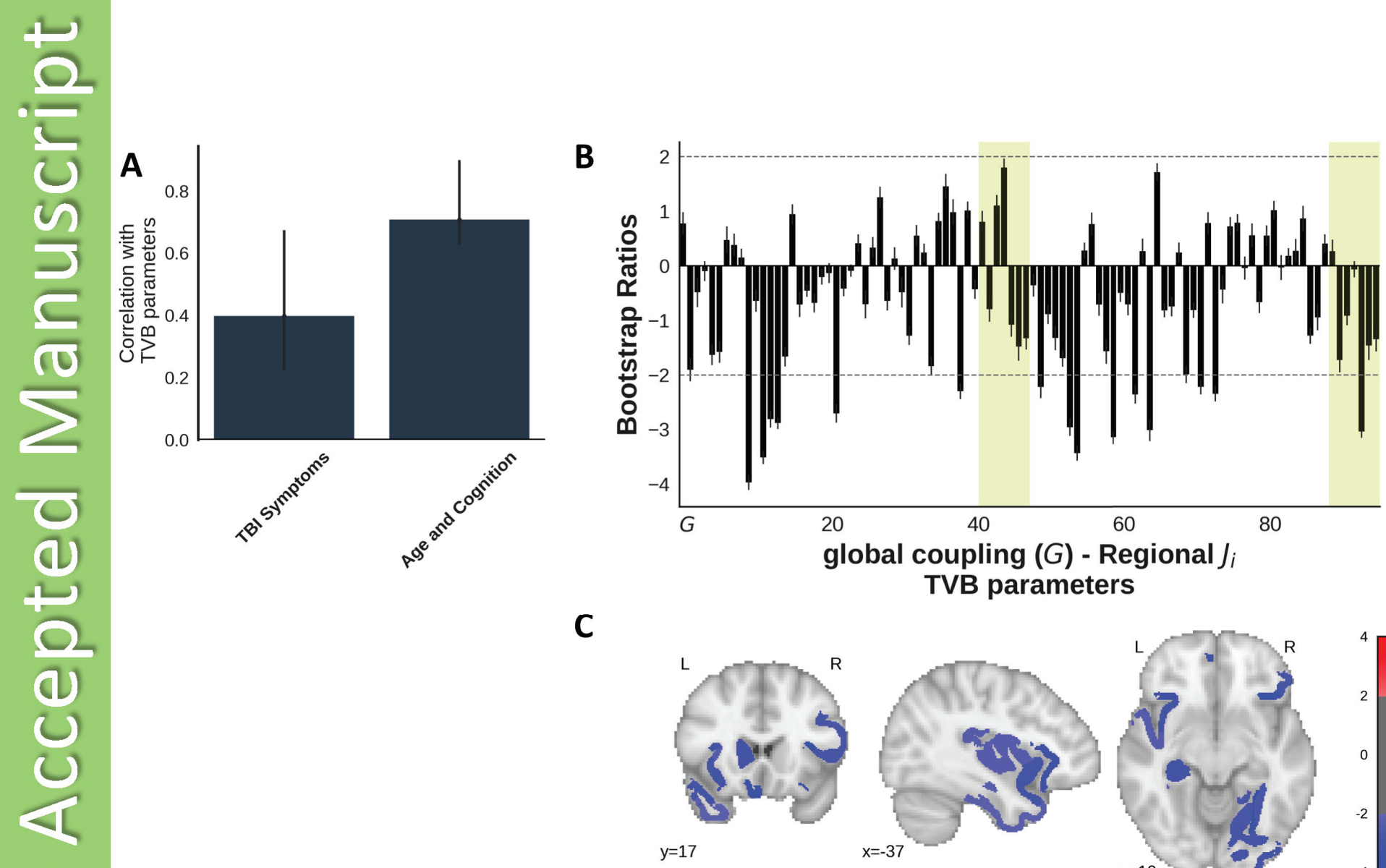

C
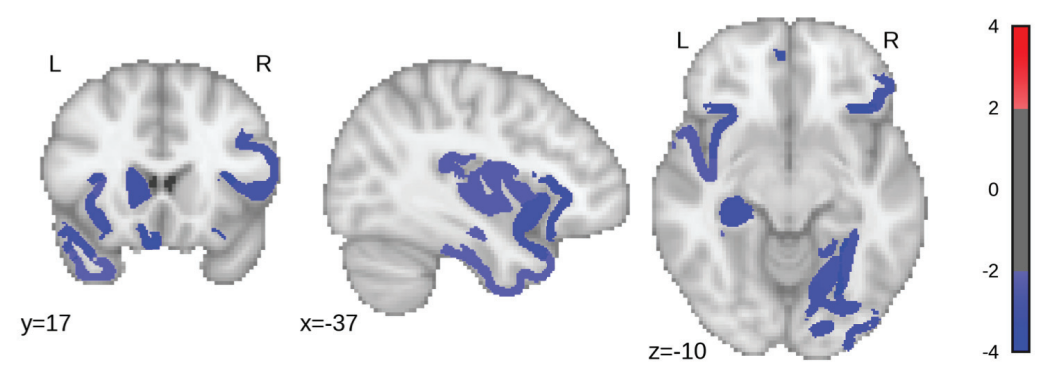

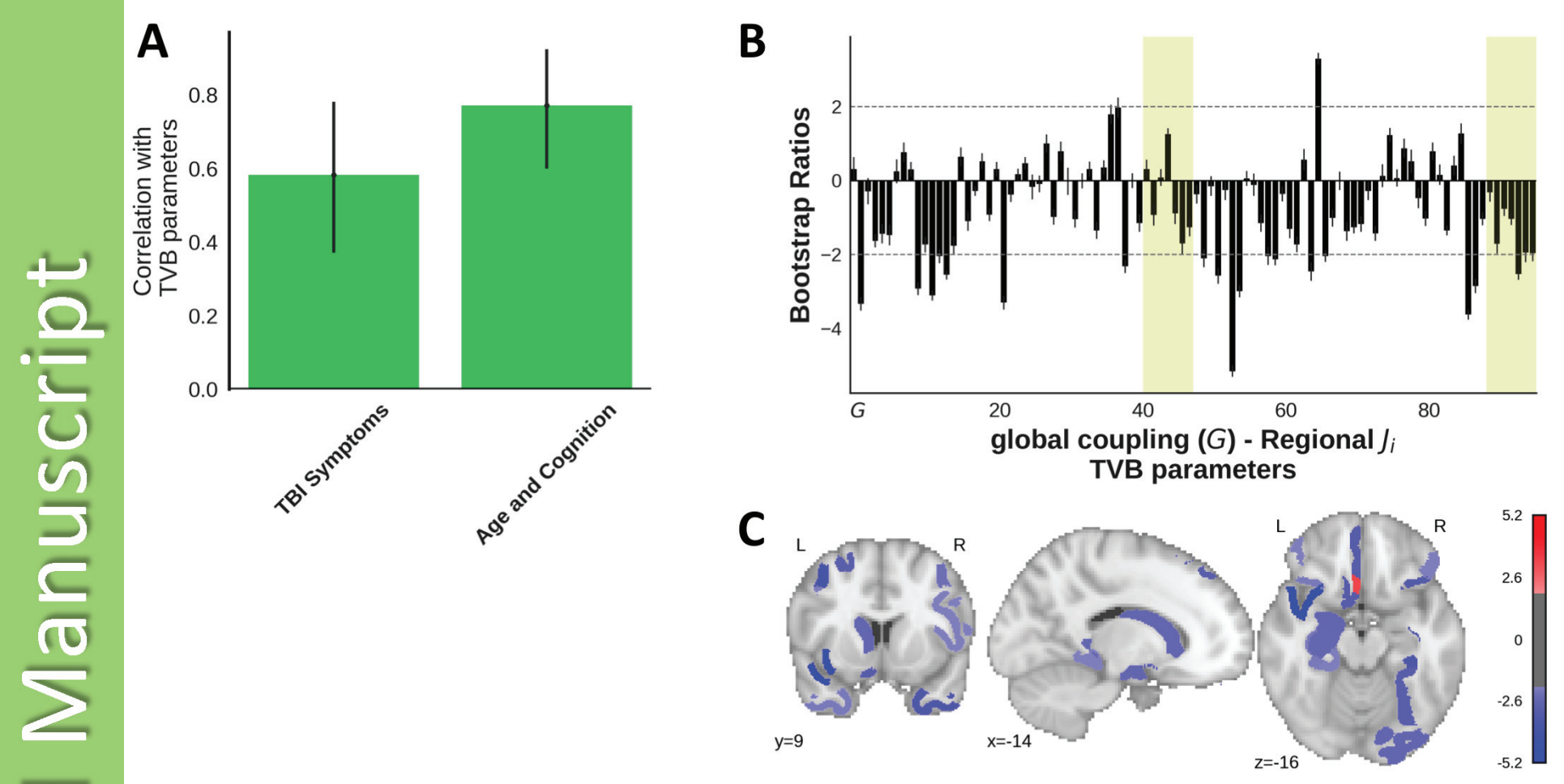

E
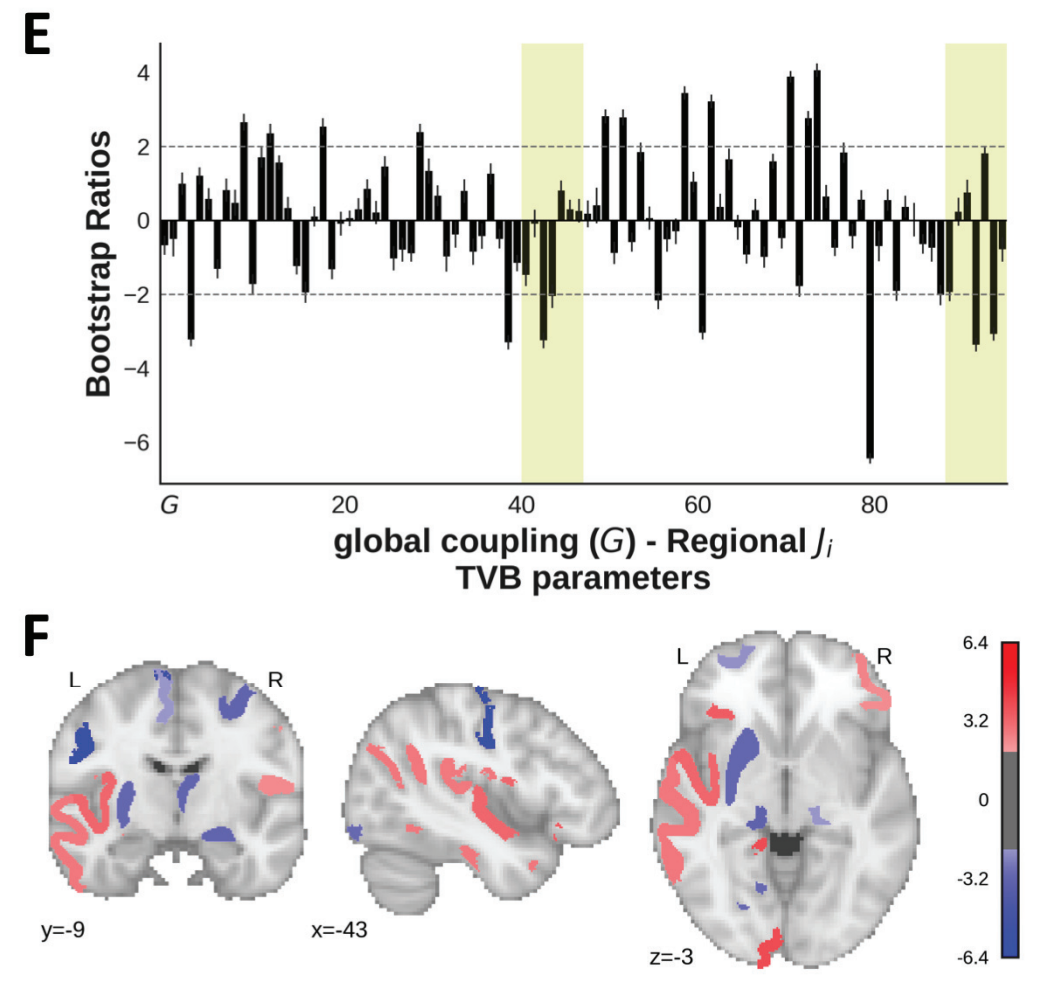